\title{
Post-stroke administration of the p75 neurotrophin receptor modulator, LM11A-31, attenuates chronic changes in brain metabolism, increases neurotransmitter levels, and improves recovery
}

Thuy-Vi V. Nguyen, Rachel H. Crumpacker, Kylie E. Calderon, Frankie G. Garcia, Jacob C. Zbesko, Jennifer B. Frye, Selena Gonzalez, Danielle A. Becktel, Tao Yang, Marco A. TaveraGarcia, Helena W. Morrison, Rick G. Schnellmann, Frank M. Longo, and Kristian P. Doyle

\section{Affiliations:}

TVN: Departments of Immunobiology and Neurology, University of Arizona, Tucson, AZ, USA

RHC: Department of Immunobiology, University of Arizona, Tucson, AZ, USA

KEC: Department of Immunobiology, University of Arizona, Tucson, AZ, USA

FGG: Department of Immunobiology, University of Arizona, Tucson, AZ, USA

JCZ: Department of Immunobiology, University of Arizona, Tucson, AZ, USA

JBF: Department of Immunobiology, University of Arizona, Tucson, AZ, USA

SG: Department of Neurology, University of Arizona, Tucson, AZ, USA

DAB: Department of Immunobiology, University of Arizona, Tucson, AZ, USA

TY: Department of Neurology and Neurological Sciences, Stanford University, Stanford, CA, USA

MATG: Department of Immunobiology, University of Arizona, Tucson, AZ, USA

HWM: College of Nursing, University of Arizona, Tucson, AZ, USA

RGS: Department of Pharmacology and Toxicology, University of Arizona, Tucson, AZ, USA, Southern Arizona VA Health Care System, Tucson, AZ

FML: Department of Neurology and Neurological Sciences, Stanford University, Stanford, CA, USA 
KPD: Departments of Immunobiology and Neurology, and the Arizona Center on Aging, University of Arizona, Tucson, AZ, USA 


\section{LM11A-31 preserves neuronal health and function after stroke \\ The Journal of Pharmacology and Experimental Therapeutics}

\section{Corresponding authors:}

Kristian P. Doyle, Ph.D.

Departments of Immunobiology and Neurology and the Arizona Center on Aging

University of Arizona, Tucson

1656 E. Mabel Street

Medical Research Building, Room 214

Tucson, AZ 85719

Telephone: (1) 520-626-8144

Email address: doylekr@email.arizona.edu

Thuy-Vi V. Nguyen, Ph.D.

Departments of Immunobiology and Neurology

University of Arizona, Tucson

1656 E. Mabel Street

Medical Research Building, Room 215

Tucson, AZ 85719

Telephone: (1) 650-269-5475

Email address: thuyvin@email.arizona.edu 
Number of text pages: 53

Number of tables: 3 (including 2 supplementary tables)

Number of figures: 10

Number of references: 54

Number of words:

226 (Abstract)

818 (Introduction)

1481 (Discussion) 


\section{List of nonstandard abbreviations:}

\begin{tabular}{|c|c|}
\hline$A \beta$ & Amyloid beta \\
\hline AD & Alzheimer's Disease \\
\hline AKT & Ak strain transforming (RAC alpha serine/threonine-protein kinase) \\
\hline APP & Amyloid precursor protein \\
\hline BDNF & Brain derived neurotrophic factor \\
\hline Cdk5 & Cyclin dependent kinase 5 \\
\hline ChAT & Choline acetyl transferase \\
\hline DH Stroke & Distal middle cerebral artery occlusion + hypoxia stroke \\
\hline dMCAO & distal Middle cerebral artery occlusion \\
\hline $\mathrm{FO}$ & Familiar object \\
\hline G-CSF & Granulocyte-colony stimulating factor \\
\hline GM-CSF & Granulocyte macrophage-colony stimulating factor \\
\hline GSH & Reduced glutathione \\
\hline GSK3 $\beta$ & Glycogen synthase kinase 3 beta \\
\hline GSSG & Oxidized glutathione \\
\hline IFN- $\gamma$ & Interferon gamma \\
\hline IL & Interleukin \\
\hline IP-10 & Interferon induced protein-10 \\
\hline IRAK & Interleukin-1 receptor associated kinase \\
\hline KC & Keratinocytes derived chemokine \\
\hline MCP-1 & Monocyte chemoattractant protein-1 \\
\hline MIP-1 $1 \alpha$ & Macrophage inflammatory protein-1 alpha \\
\hline MIP-1 $\beta$ & Macrophage inflammatory protein-1 beta \\
\hline MIP-2 $\alpha$ & Macrophage inflammatory protein-2 alpha \\
\hline
\end{tabular}




\begin{tabular}{|c|c|}
\hline NeuN & Neuronal nuclei \\
\hline$N F \kappa B$ & Nuclear factor kappa-light-chain-enhancer of activated B cells \\
\hline NGF & Nerve growth factor \\
\hline NMDA & N-methyl-D-aspartate \\
\hline NO & Novel object \\
\hline NOR & Novel object recognition \\
\hline OF & Open field \\
\hline PCA & Principal component analysis \\
\hline$P 75^{\mathrm{NTR}}$ & p75 neurotrophin receptor \\
\hline PI3K & Phosphoinositide 3 kinase \\
\hline RANTES & Regulated on activation normal $\mathrm{T}$ cell expressed and secreted \\
\hline $\mathrm{RI}$ & Recognition index \\
\hline SAB & Spontaneous alternation behavior \\
\hline TCA & Tricarboxylic acid \\
\hline $\mathrm{TH}$ & Tyrosine hydroxylase \\
\hline Thy1 & Thymocyte differentiation antigen 1 \\
\hline TNF- $\alpha$ & Tumor necrosis factor-alpha \\
\hline TPA & Tissue plasminogen activator \\
\hline
\end{tabular}

\section{Recommended section assignment:}

Drug Discovery and Translational Medicine 


\section{ABSTRACT}

The aim of this study was to test whether post-stroke oral administration of a small molecule p75 neurotrophin receptor ( $p 75^{\mathrm{NTR}}$ ) modulator (LM11A-31) can augment neuronal survival and improve recovery in a mouse model of stroke. Mice were administered LM11A-31 for up to 12 weeks, beginning 1 week after stroke. Metabolomic analysis revealed that after 2 weeks of daily treatment, mice that received LM11A-31 were distinct from vehicle treated mice by principal component analysis and had higher levels of serotonin, acetylcholine, and dopamine in their ipsilateral hemisphere. LM11A-31 treatment also improved redox homeostasis by restoring reduced glutathione. It also offset a stroke induced reduction in glycolysis by increasing acetylCoA. There was no effect on cytokine levels in the infarct. At 13 weeks following stroke, adaptive immune cell infiltration in the infarct was unchanged in LM11A-31 treated mice, indicating that LM11A31 does not alter the chronic inflammatory response to stroke at the site of the infarct. However, LM11A-31 treated mice had less brain atrophy, neurodegeneration, tau pathology, and microglial activation in other regions of the ipsilateral hemisphere. These findings correlated with improved recovery of motor function on a ladder test, improved sensorimotor and cognitive abilities on a nest construction test, and less impulsivity in an open field test. These data support small molecule modulation of the $\mathrm{p} 75$ neurotrophin receptor for preserving neuronal health and function during stroke recovery.

\section{SIGNIFICANCE STATEMENT}

The findings from this study introduce the p75 neurotrophin receptor as a novel small molecule target for promotion of stroke recovery. Given that LM11A-31 is in clinical trials as a potential therapy for Alzheimer's disease, it could be considered as a candidate for assessment in stroke or vascular dementia studies. 


\section{INTRODUCTION}

People over the age of 55 have a 1 in 6 lifetime risk of having a stroke (Seshadri et al., 2006) and there are more than 6.5 million survivors of stroke in the United States (US) (http://www.strokecenter.org/patients/about-stroke/stroke-statistics/; Donkor, 2018). The only FDA approved drug available for the treatment of stroke is tissue plasminogen activator (TPA), but it must be administered within 4.5 hours of ischemic stroke onset. Mechanical thrombectomy can also be used to restore cerebral blood flow in acute ischemic stroke, however, less than $20 \%$ of patients qualify for this type of reperfusion therapy and it must be initiated within 24 hours of stroke onset (Rabinstein, 2020). In contrast, no pharmacologic therapies are available to enhance recovery in the days and weeks after stroke. This prompts the need to identify compounds commonly applied to, or in the pipeline for, the treatment of other neurological disorders to determine if they can promote neural repair and recovery of cellular function after stroke. One such example is LM11A-31, a small molecule ligand for the p75 neurotrophin receptor (p75 ${ }^{\mathrm{NTR}}$ ). LM11A-31 is currently under Phase Ila clinical evaluation for the treatment of mild to moderate Alzheimer's disease (AD; ClinicalTrials.gov Identifier: NCT03069014).

p75 ${ }^{\text {NTR }}$ is expressed in basal forebrain cholinergic, cortical, hippocampal, noradrenergic, and other central nervous system (CNS) neurons (Becker et al., 2018) that have critical roles in learning and memory, cognition, psychological wellbeing, and sensorimotor functions. It is a tumor necrosis factor (TNF) family member receptor that recognizes neurotrophins, including nerve growth factor, neurotrophin-3, neurotrophin-4/5, and brain derived neurotrophic factor (BDNF). Although once categorized as a death initiating receptor, it is now clear that it can also promote cell survival through phosphoinositide 3-kinase (PI3K)/protein kinase B (AKT) and interleukin-1 receptor-associated kinase (IRAK)/nuclear factor kappa B (NFאB) dependent signaling mechanisms (Longo and Massa, 2008). The balance of pro-survival versus pro-apoptotic 
signaling is determined by the availability of adaptor molecules, which is itself determined by cell type and contextual cues (Longo and Massa, 2008).

LM11A-31 specifically functions as a $\mathrm{p} 75^{\mathrm{NTR}}$ ligand. This specificity is supported by multiple lines of evidence, including the rapid recruitment of the IRAK p $75^{\text {NTR }}$ intracellular adaptor following treatment in vitro, the ability of LM11A-31 to block a p $75^{\mathrm{NTR}}$ antibody from binding to the receptor, and conversely the ability of p75 NTR antibodies to block LM11A-31 activity. It is also supported by loss of ligand signaling and function in $\mathrm{p} 75^{\mathrm{NTR}-/-}$ neurons, nerve growth factor (NGF) like promotion of $p 75^{\text {NTR }}$ endocytosis, and no detectable effect of LM11A-31 on tropomyosin receptor A (TrkA) (Longo and Massa, 2008; Longo and Massa, 2013). At nanomolar concentrations in vitro, LM11A31 prevents neuronal death, promotes neurotrophic signaling, and inhibits neurodegenerative signaling through the p75 NTR (Massa et al., 2006; Yang et al., 2008). Therefore, LM11A-31 functions as a $\mathrm{p} 75^{\mathrm{NTR}}$ receptor modulator rather than a simple agonist or antagonist, switching p75 NTR from neurodegenerative to neurotrophic signaling in neurons expressing p75 NTR (Xie et al., 2019).

Importantly, LM11A-31 has shown therapeutic effects in multiple animal models of neurological diseases, including post-traumatic brain injury (Shi et al., 2013), human immunodeficiency virus (HIV) related dementia (Meeker et al., 2016; Fogle et al., 2021; Xie et al., 2021), contusion spinal cord injury (Tep et al., 2013), Huntington's disease (Simmons et al., 2016; Simmons et al., 2021), and AD (Knowles et al., 2013; Nguyen et al., 2014; Simmons et al., 2014; Yang et al., 2020a). In $A D, L M 11 A-31$ has been shown to be efficacious in male and female mice and in two different mouse models of AD (Simmons et al., 2014). It has also been shown to reverse neuronal degeneration in late stage AD pathology in transgenic mice (Knowles et al., 2009; Knowles et al., 2013; Simmons et al., 2013; Nguyen et al., 2014; James et al., 2017). LM11A-31 is orally bioavailable and CNS penetrant, exerting maximal efficacy in the $25-50 \mathrm{mg} / \mathrm{kg}$ range $(\mathrm{HCl}$ salt 
form), which at $50 \mathrm{mg} / \mathrm{kg}$ corresponds to a human equivalent dose of $284 \mathrm{mg}$ for a $70 \mathrm{~kg}$ adult, per body surface area mouse to human conversion (Massa et al., 2006; Yang et al., 2008). LM11A-31 reaches and preferentially accumulates in the brain compared to blood (Knowles et al., 2013), which reduces the likelihood of systemic toxicity.

In light of the efficacy of LM11A-31 across a wide spectrum of neurological diseases, its attractive pharmacokinetic properties, and the fact that it is currently undergoing clinical testing for the treatment of $A D$, we sought to determine whether LM11A-31 could protect neurons from the chronic sequelae of stroke that cause neurodegeneration in the weeks after stroke. We discovered in a mouse model of experimental stroke that daily oral administration of LM11A-31 can mitigate secondary neurodegeneration, increase neurotransmitter levels, and improve functional recovery. These findings correlated with changes in redox homeostasis and energy metabolism in the injured hemisphere, but no changes in inflammation within the infarct. Together, these data support the use of LM11A-31 for preserving neuronal health and function in patients recovering from stroke.

\section{MATERIALS AND METHODS}

\section{Mice}

17-18-month-old, male C57BL/6J (The Jackson Laboratory, Stock No: 000664) mice were used for all experiments. All mice were given ad libitum access to food and water and were maintained in a 12-hour light/dark cycle in a temperature controlled housing facility. In accordance with Stroke Therapy Academic Industry Roundtable (STAIR) recommendations (Lyden et al., 2021), mice from Phase I (Cohort 1) were randomly allocated into treatment groups before the stroke/sham 
procedure. Additionally, mice from Phase II (Cohort 2) were stroked and stratified into treatment groups based on Day 2 post-stroke ladder rung data. All dosing, behavioral experiments, imaging, and morphological and biochemical analyses were performed with blinding relative to vehicle versus LM11A-31 treatment. All procedures met National Institute of Health guidelines with the approval of the University of Arizona Institutional Animal Care and Use Committee.

\section{Stroke and sham surgeries}

Distal Middle Cerebral Artery Occlusion (dMCAO) with hypoxia (DH stroke) was performed as previously published (Doyle et al., 2012). Briefly, following anesthesia by isoflurane inhalation, the temporalis muscle was exposed by incision. The right MCA was identified and exposed using a microdrill on the skull. The meninges were cut, and the MCA was cauterized. The incision was then closed using surgical glue. Directly after receiving the dMCAO, mice underwent hypoxia ( $9 \%$ oxygen and $91 \%$ nitrogen) for 45 minutes. Sham operated mice underwent the same surgical steps, except for cauterization of the dMCA. They were also given $9 \%$ oxygen and $91 \%$ nitrogen for 45 minutes immediately after surgery. Throughout the surgery and hypoxia, mouse core temperature was monitored and maintained at $37^{\circ} \mathrm{C}$. The purpose of hypoxia in this model is to increase infarct size, as C57BL/6 mice that receive a dMCAO without hypoxia have significantly smaller infarcts (Doyle et al., 2012). This stroke model results in a consistent infarct volume of approximately $25 \%$ of the ipsilateral hemisphere located in the somatosensory cortex and the M1 region of the motor cortex (Doyle et al., 2012; Nguyen et al., 2018). Mice were euthanized at 3 and 13 weeks following surgery with isoflurane inhalation and exsanguination and were perfused intracardially with $0.9 \%$ saline without heparin.

\section{LM11A-31}


LM11A-31 [2-amino-3-methyl-pentanoic acid (2-morpholin-4-yl-ethyl)-amide], an isoleucine derivative, was synthesized by Ricerca Biosciences at $>97 \%$ purity, as assessed by liquid chromatography/mass spectroscopy. LM11A-31 (-31; final dose: $50 \mathrm{mg} / \mathrm{kg}, \mathrm{HCl}$ salt form) was prepared in sterile water and administered by oral gavage. This dose is efficacious in both male and female mice, in two models of $A D$, and has high efficacy on mid- to late-stage $A D$ progression (Knowles et al., 2013; Nguyen et al., 2014; Simmons et al., 2014). An equivalent volume (10 $\mu$ l per gram of body weight) of sterile water was administered by oral gavage to vehicle (veh) treated mice.

\section{Global metabolic profiling}

At 3 weeks following surgery, infarcts from stroked mice, and the equivalent region of the cortex from sham operated mice, were resected and the remainder of the ipsilateral hemispheres were snap frozen and sent to Metabolon (Morrisville, NC) for processing and analysis (Ribbenstedt et al., 2018). At Metabolon, samples were prepared by first removing proteins and extracting small molecules and metabolites through a series of aqueous and organic extractions. The resulting extract was divided into 4 aliquots, each of which then underwent metabolic profiling. Two aliquots were profiled using reverse phase ultrahigh performance liquid chromatography/tandem mass spectrometry (UPLC-MS/MS) in acidic positive ion conditions, 1 aliquot in conditions optimized for more hydrophobic compounds and the other in conditions optimized for more hydrophilic compounds. Another aliquot was profiled using UPLC-MS/MS in basic negative ion conditions, and the $4^{\text {th }}$ aliquot using hydrophilic interaction ultra-performance liquid chromatography (HILIC/ULPC-MS/MS). Data extraction, compound identification, metabolite quantification, and data normalization were performed using Metabolon's proprietary software. Quality control and curation procedures were used to remove artifacts, mis-assignments, and background noise (Choi et al., 2018). 


\section{Multiplex immunoassay}

At 3 weeks following surgery, infarcts from stroked mice and the equivalent region of the cortex from sham operated mice were dissected and sonicated in ice cold $0.1 \mathrm{M}$ phosphate buffered saline containing $1 \%$ triton $\mathrm{X}-100$ and $0.1 \%$ sodium deoxycholate, Protease Inhibitor Cocktail (1:100; Millipore Sigma), and Phosphatase Inhibitor Cocktail 2 (1:100; Millipore Sigma). Following centrifugation, the total protein concentration of each supernatant was measured using a Direct Detect Infrared Spectrometer (Millipore Sigma). Cytokines/chemokines were then quantified by multiplex immunoassay using a high sensitivity mouse multiplex magnetic bead kit (Millipore Sigma) and performed according to the manufacturer's recommendations (MILLIPLEX MAP Mouse Cytokine/Chemokine Magnetic Bead Panel-Immunology Multiplex Assay, MCYTOMAG70K). Each lysate sample, standard, and quality control were measured in duplicate. Plates were read using a MAGPIX instrument (Luminex, Austin, TX), and results were analyzed using MILLIPLEX Analyst 5.1 software (Millipore Sigma). For those analytes that were below the lower limit of detection, the lower limit of detection values was used for data analysis. Concentrations of cytokines/chemokines were normalized to concentrations of total protein in the lysates.

\section{Processing brain tissue for histology and immunohistochemistry}

At 13 weeks following stroke, brains were post-fixed in $4 \%$ paraformaldehyde for 24 hours, followed by cryopreservation in $30 \%$ sucrose. Frozen coronal sections (40 $\mu \mathrm{m})$ were then taken through the entire brain using a freezing Microtome HM 450 sliding microtome (ThermoFisher Scientific, Waltham, MA). Brain sections were sequentially placed into 16 microcentrifuge tubes and stored in cryoprotectant medium at $-20^{\circ} \mathrm{C}$ until staining. 


\section{Nissl staining}

For Nissl staining, sections were mounted, dehydrated and cleared in xylenes, rehydrated, immersed in $0.5 \%$ cresyl violet acetate (MilliporeSigma, Burlington, MA) solution for 1 minute, rinsed with distilled water, differentiated in $0.25 \%$ acetic alcohol, dehydrated though a graded ethanol series, cleared, and coverslipped with mounting media.

\section{Immunohistochemistry}

For immunostaining, sections were immunostained according to standard protocols (Nguyen et al., 2014). The following antibodies were used: mouse anti-neuronal nuclei (NeuN; Millipore Sigma), goat anti-choline acetyl transferase (ChAT; Millipore Sigma), rabbit anti-tyrosine hydroxylase (TH; Millipore Sigma), mouse anti-AT8 [recognizes tau phosphorylated at serine 202 and threonine 205 ( $p$-tauSer202/Thr205; ThermoFisher Scientific)], rat anti-CD68 (Bio-Rad AbD Serotec, Hercules, CA), rat anti-mouse B220/CD45R biotinylated (BD Biosciences, San Jose, $\mathrm{CA})$, and hamster anti-CD3e (BD Biosciences). Briefly, free floating sections were immunolabeled with antibodies (1:500 for NeuN, 1:600 for ChAT, 1:1000 for TH, 1:800 for AT8, 1:1000 for CD68, 1:500 for B220/CD45R, and 1:1000 for CD3e) in conjunction with Mouse on Mouse (M.O.M.) Detection kit (for AT8), ABC Vector Elite, and 3,3'-diaminobenzidine (Vector Laboratories, Burlingame, CA) for visualization.

\section{Microscopy and imaging}

Microscopy was performed by an experimenter blinded to treatment group. Captured images were analyzed by an independent experimenter also blinded to treatment group. Images shown in the figures are representative images that best depict the data represented by the graphs. Images 
were captured with a Keyence (Elmwood Park, NJ) BZ-X700 digital microscope or a Leica (Buffalo Grove, IL) DM6000B light microscope coupled with a Leica DFC 7000 T camera. The Mouse Brain in Stereotaxic Coordinates of Franklin and Paxinos $3^{\text {rd }}$ edition (Franklin and Paxinos, 2008) was used as an anatomical reference atlas for mouse brain structures and Bregma coordinates.

\section{Quantitative analysis of brain atrophy}

A $2 \times$ objective was used to visualize brain atrophy following stroke. To measure brain atrophy, 2 sections per mouse, at Bregmas $-1.70 \mathrm{~mm}$ and $-1.94 \mathrm{~mm}$ were analyzed for anatomical abnormalities via Nissl staining. The area of the lateral ventricles and the thickness of the primary somatosensory cortex, from the corpus callosum through layers I-VI of the cortex of each hemisphere were manually traced for each section. The area of the ventricles and the thickness of the cortex were computed using NIH Image J software (Media Cybernetics, Rockville, MD).

\section{Quantitative analysis of neuronal degeneration}

For quantitative analysis of of $\mathrm{NeuN}+$ immunoreactivity, $\mathrm{NeuN}+$ immunolabeled neurons in the primary somatosensory cortex adjacent to the infarct, and in the area of axonal degeneration in thalamic white matter regions were measured in 2 sections for each mouse. Section 1 was at the level of Bregma $+1.18 \mathrm{~mm}$, and section 2 was at the level Bregma $+0.74 \mathrm{~mm}$. Using a $10 \times$ objective, 2 fields were randomly selected from the ipsilateral cortex and thalamus for each section. Corresponding fields were taken from the contralateral hemisphere. For measurement of cholinergic neurodegeneration, ChAT+ immunolabeled cholinergic somas and their neurites in the medial septum of the basal forebrain were imaged. The total amount of ChAT+ immunoreactivity in the medial septum was quantified in 2 sections for each mouse with section 
1 at Bregma $+1.18 \mathrm{~mm}$, and section 2 at Bregma $+0.50 \mathrm{~mm}$. For measurement of $\mathrm{TH}+$ fiber projections, 2 fields were randomly selected from the primary somatosensory cortex adjacent to the infarct using a $10 \times$ objective. Three sections were analyzed per mouse for a total of 6 fields per mouse between Bregma $+1.18 \mathrm{~mm}$ and Bregma $+0.74 \mathrm{~mm}$. Corresponding fields were taken from the contralateral hemisphere. The percent area occupied by $\mathrm{NeuN}+, \mathrm{ChAT}+$, and $\mathrm{TH}+$ staining in each region was computed using $\mathrm{NIH}$ Image $\mathrm{J}$ software.

\section{Quantitative analysis of tau immunoreactivity}

AT8/p-tau+ immunoreactivity in the degenerating white matter tracts of the thalamus were visualized with a $10 \times$ objective. In this analysis, 3 sections that contained the thalamus were selected; section 1 contained the thalamus at Bregma $-0.70 \mathrm{~mm}$, section 2 contained the thalamus at Bregma $-1.46 \mathrm{~mm}$, and section 3 contained the thalamus at Bregma $-2.18 \mathrm{~mm}$ for each mouse. The total percentage occupied by $p$-tau+ staining in the thalamus on each section was computed using NIH Image $\mathrm{J}$ software. Sections from APP (amyloid precursor protein) (Nguyen et al., 2014; Nguyen et al., 2018) mutant mice displaying both amyloid and tau pathologies were used as a positive control for the pTau+ staining with the AT8 antibody.

\section{Quantitation of CD68, B220/CCD45R, and CD3e immunoreactivity in the stroke infarct}

Immunoreactivity of CD68+ labeled microglia/macrophages, B220+ labeled B cells, and CD3e+ labeled T cells were visualized with a 10x objective and measured in the infarct using NIH Image J (Media Cybernetics, Rockville, MD). Three to 6 sections were stitched from 3-4 fields to capture the entire infarct area between Bregma $-0.46 \mathrm{~mm}$ and Bregma $-1.94 \mathrm{~mm}$ per mouse. 


\section{Quantitation of CD68 and CD3e immunoreactivity in white matter tracts of the thalamus}

CD68+ microglia and macrophages and CD3e+ T cells were visualized with a $10 \times$ objective and analyzed separately per mouse at Bregma -1.70 in the white matter tracts of the thalamus (area of axonal degeneration) following stroke surgery. Two non-overlapping, adjacent fields were randomly selected from 2 sections per mouse for CD68+ quantification from each hemisphere of the thalamus at the reticular thalamus nucleus to the ventral posteromedial thalamus nucleus. One field ( 2 sections per mouse for a total of 2 fields) was taken for CD3e+ quantification at the same brain region. The percentage occupied by $\mathrm{CD68}+$ or $\mathrm{CD} 3 \mathrm{e}+$ immunostaining was computed.

\section{Ladder rung test}

The ladder rung test was adapted from the ladder rung walking task (Rha et al., 2011; Doyle et al., 2012). The apparatus consisted of plexiglass rungs $(10.16 \mathrm{~cm}$ length $\times 0.32 \mathrm{~cm}$ diameter $)$ spaced at a constant distance of $0.64 \mathrm{~cm}$ apart, inserted between two plexiglass walls $(0.64 \mathrm{~cm}$ width $\times 76.20 \mathrm{~cm}$ length $\times 15.24 \mathrm{~cm}$ height) and spaced $3.18 \mathrm{~cm}$ apart, providing a wide enough space for the animal to transverse the ladder. The apparatus was suspended $18 \mathrm{~cm}$ from the tabletop surface using blocks. The test was performed in a darkened room with a desk lamp illuminating the start zone to incentivize the animals to move across the ladder, and a small box was placed at the end zone. The mice were kept in a plastic chamber $(9 \mathrm{~cm} \times 8 \mathrm{~cm} \times 14 \mathrm{~cm})$ that was level with the plexiglass rungs at the start zone. As a mouse moved across the ladder, its steps were recorded from below, using a camera that slides along the surface of the testing table. Prior to pre-surgery testing, training was necessary to ensure that the animals were able to spontaneously traverse the apparatus. Eight training trials were required for animals to make an 
acceptable maximum number of limb placement errors $(\leq 10-12 \%)$. Each mouse was tested once on each test day. The ladder apparatus was cleaned thoroughly with $10 \%$ ethanol and wiped dry between trials to minimize olfactory cues. Video recordings of each mouse traversing the ladder were analyzed at $0.25-0.30 \times$ playback speed using standard film playback software (VLC media player for Mac OSX). A trained observer focused on the front limb on the side of the body contralateral to the stroke infarct (i.e., front left paw). Once the mouse placed all four limbs on the ladder, the observer began counting correct steps and missteps of the front left paw. If the paw was placed on a rung and was not removed until the following step, a correct step was recorded. If the paw was placed on a rung and then slipped off the rung, or if the paw missed the rung entirely, a misstep was recorded. Two trained experimenters scored each video, and their values were averaged. The percent correct foot placement was calculated as $100 \times$ (number of correct steps/number of correct steps + number of missteps).

\section{Nest construction test}

Mice were transferred to individual testing cages with all environmental enrichment items (i.e., domes, tunnels) removed. One square $(5 \mathrm{~cm})$ piece of nestlet (Ancare, Bellmore, NY) made of pressed cotton batting, was placed in the same place of each test cage. Mice were provided with food and water. Testing was performed overnight, and images of nests built by the mice were captured the next morning. Nests were scored as follows on a scale of 1-5 based on how they were constructed: (i) nestlet was largely untouched (>90\% remaining intact; 1 point); (ii) nestlet was partially torn up (50-90\% remaining intact; 2 points); (iii) nestlet was mostly shredded, but often there was no identifiable nest site $(<50 \%$ of the nestlet remaining intact and $<90 \%$ within a quarter of the cage floor area, i.e., the cotton was not gathered into a nest, but scattered about the cage; 3 points); (iv) an identifiable, but flat nest (>90\% of the nestlet shredded and the material 
was gathered into a nest within a quarter of the cage floor area, but the nest was flat, with walls higher than the mouse's body height around $<50 \%$ of its circumference; 4 points); and ( $v$ ) a near perfect nest $(>90 \%$ of the nestlet shredded and shaped into a crater, with walls higher than the mouse's body height around $>50 \%$ of its circumference; 5 points). This nest construction scoring system is based on Deacon and colleagues' protocol (Deacon, 2006). Two trained experimenters scored each nest, and their values were averaged.

\section{Y-maze spontaneous alternation behavior (SAB) test}

Mice were tested in a Y-shaped maze composed of beige acrylonitrile butadiene styrene (ABS) plastic and consisting of two symmetrical arms and one longer arm set at a $120^{\circ}$ angle from each other (equal arms: $7.5 \mathrm{~cm}$ width $\times 37.0 \mathrm{~cm}$ length $\times 12.5 \mathrm{~cm}$ height; longer arm: $7.5 \mathrm{~cm}$ width $\times$ $42.0 \mathrm{~cm}$ length $\times 12.5 \mathrm{~cm}$ height). Mice were placed at the end of the longest arm of the maze, facing outward, and allowed to freely explore the 3 arms for 5 minutes. Over the course of multiple entries, mice normally exhibit a tendency to visit a new arm of the maze rather than visiting a recently visited arm. An entry was recorded when all four limbs of the mouse were within an arm of the maze. The number of arm entries and the number of triads were recorded and scored by the ANY-maze behavioral video tracking software (Stoelting, Wood Dale, IL), and the percentage of SAB was calculated by the software. The Y-maze apparatus was cleaned with $10 \%$ ethanol and wiped dry between trials to minimize olfactory cues.

\section{Novel object recognition (NOR) test}


The NOR arena consisted of a white opaque box measuring $38.0 \mathrm{~cm}$ width $\times 48.0 \mathrm{~cm}$ length $\times$ $20.0 \mathrm{~cm}$ height made of ABS plastic. Mice were habituated to the NOR arena over the course of 2 days prior to testing day. During the first day of habituation, mice were placed with cage mates for 15 minutes in the NOR arena. The following day, each mouse was allowed to explore the arena alone for 15 minutes. On testing day, each mouse first underwent an "object familiarization phase," during which they were placed in the arena along with two identical objects located at different corners of the arena $5 \mathrm{~cm}$ from the walls. The objects consisted of children's building blocks of different shapes and colors. Mice were habituated to the NOR arena over the course of 2 days prior to testing day. During the first day of habituation, mice were placed with cage mates for 15 minutes in the NOR arena. The following day, each mouse was allowed to explore the arena alone for 15 minutes. On testing day, each mouse first underwent an "object familiarization phase", during which they were Published studies confirmed no innate preference between objects. Mice were allowed to explore the arena and objects for 5 minutes. Object exploration was defined as contact with the object by the mouse's nose within $2 \mathrm{~cm}$ of the object, which was recorded by ANY-maze behavioral video tracking software 4 hours following the object familiarization phase, each mouse underwent an "object recognition testing phase", during which they were placed back in the arena with one of the objects they previously explored during the familiarization phase [the familiar object (FO)], and a novel object (NO). The object role (novel versus familiar) and position (left versus right) were balanced within each experimental group. Different sets of objects were used for each timepoint to control for biases from previously exposed objects. ANY-maze behavioral video tracking software was used to compute the time each mouse spent exploring each object. The recognition index (RI) was calculated as (NO Time)/(NO Time + FO Time) and expressed as a percentage by multiplying by 100 . The NOR apparatus was cleaned with $10 \%$ ethanol between trials to minimize olfactory cues. 


\section{Open field (OF) testing}

The OF test assesses exploratory habits in a relatively large novel environment. The OF apparatus consisted of a beige ABS plastic arena measuring $50 \mathrm{~cm}$ length $\times 50 \mathrm{~cm}$ width $\times 50 \mathrm{~cm}$ height. The arena was divided into $10 \mathrm{~cm}$ squares (grid). The center zone of the arena measured $30 \mathrm{~cm}$ length $\times 30 \mathrm{~cm}$ width. The periphery zone included all the squares along the edge of the arena and measured $8 \mathrm{~cm}$ width. The space between the center and periphery zones was considered the "dead" zone. Each mouse was placed into the arena and allowed to explore for 5 minutes. During these 5 minutes, ANY-maze software was used to track the center of the animal's body within the arena. The software then computed the number of entries and exits into each zone for each mouse. The OF apparatus was cleaned with $10 \%$ ethanol and wiped dry between trials to minimize olfactory cues.

\section{Power analysis and sample size calculations}

Sample sizes were determined by a priori power analysis with data from our pilot studies and previous published studies (Nguyen et al., 2014; Nguyen et al., 2016; Nguyen et al., 2018). For the metabolomics and multiplex immunoassay analyses (Phase I of the study), according to the calculations, the minimum number of mice needed to attain statical significance of $p<0.05$ with an $80 \%$ probability was $n=5$ mice/experimental group when we anticipated a $\geq 20 \%$ difference between experimental groups with an SD of $11 \%$. For the behavioral studies (Phase II), the minimum number of mice needed to attain statistical significance of $p<0.05$ with an $80 \%$ probability was $n=9$ mice/experimental group when we anticipated $a \geq 20 \%$ difference between experimental groups with an SD of $15 \%$. Therefore, we included a total of 10 male mice in each 
experimental group to account for possible deaths due to age or surgery for behavior tests. All the mice used for behavior tests were also used for morphological studies (Phase II of the study), even though the minimum number of mice needed to attain statistical significance of $p<0.05$ with an $80 \%$ probability was $n=9$ mice/experimental group when we anticipated a $\geq 20 \%$ difference between experimental groups with an SD of $10 \%$ for this analysis.

\section{Statistics}

Data are presented as mean \pm SD. Datasets containing 3 groups were analyzed using a one-way ANOVA, followed by post-hoc Newman-Keuls testing. Datasets containing 2 groups were evaluated by Student's t-test. Significance was set at $p<0.05$. For all figures, ${ }^{*} p$ is $<0.05,{ }^{* *} p$ is $<0.01,{ }^{* *} p$ is $<0.001$, and ${ }^{* * *} p$ is $<0.0001$. The statistical summary of the metabolomics data is shown in Table 1. Metabolon's proprietary Pathway Surveyor tool was used to identify key pathways significantly altered by stroke and LM11A-31 treatment.

\section{RESULTS}

\section{Study design}

The goal of this study was to evaluate the therapeutic effect of a p75 NTR small molecule ligand on recovery from stroke. To achieve this goal, the study was divided into two testing phases. For Phase I (Figure 1A, Study design), mice were randomized and underwent stroke or sham surgery. A week after surgery, mice were orally administered LM11A-31 for 7 days per week, one dose per day, for 2 weeks, beginning 1 week after surgery (experimental groups: $n=5$ for shamveh, $n=5$ for stroke-veh, and $n=6$ for stroke-31). At 3 weeks following surgery, mice were 
euthanized, and their infarcts were processed for multiplex immunoassay. The remainder of their ipsilateral hemispheres was processed for metabolomics. The goal of Phase I was to evaluate how LM11A-31 alters brain biochemistry and post-stroke inflammation during treatment.

For Phase II (Figure 1B, Study design), a separate cohort of mice underwent baseline ladder rung testing, and their scores were used to stratify mice into either LM11A-31 or vehicle treatment groups (experimental groups: $n=10$ for stroke-veh, and $n=10$ for stroke-31). Mice then underwent stroke surgery and were administered with either LM11A-31 or vehicle for 5 days per week, one dose per day, by oral administration for 12 weeks, beginning 1 week after surgery. The ladder rung test was repeated at 2 days, 2 weeks, 6 weeks, and 10 weeks after stroke surgery. During the 12th week of treatment, mice were tested on a panel of behavioral tests, including a nest construction test, a Y-maze Spontaneous Alternation Behavior (SAB) test, a Novel Object Recognition (NOR) test, and an Open Field (OF) test. After 12 weeks of treatment (13 weeks post-stroke), mice were euthanized, and their brains were processed for histology and immunostaining. The goal of Phase II was to evaluate treatment efficacy of LM11A-31 in the context of stroke recovery.

\section{Phase I: Global metabolic profiling of LM11A-31 treated mice}

After 2 weeks of treatment ( 3 weeks post-stroke or sham surgery), global metabolic profiling of the ipsilateral hemispheres from the mice treated with LM11A-31 and vehicle returned measurements for 645 compounds. Following log transformation and imputation of missing values with the minimum observed value for each compound, a three-way ANOVA was performed to identify the chemicals that were significantly different between experimental groups. 
Among the mice that were treated with vehicle alone, 169 chemicals were present at different signal intensities in stroked mice compared to sham operated mice. Among the mice that were treated with LM11A-31, 135 chemicals were present at different intensities in stroked compared to sham operated mice. The least difference in metabolic profile, which equated to changes in 38 chemicals, was detected among mice that underwent sham surgery, between those treated with LM11A-31 and those treated with vehicle. However, the equivalent comparison among stroked mice showed 159 chemicals that were altered by treatment with LM11A-31. These data indicate that (i) there are substantial alterations in brain metabolism at 3 weeks following stroke; (ii) that treatment with LM11A-31 in sham operated mice does not substantially alter brain metabolism; and (iii) that 2 weeks of LM11A-31 versus vehicle administration substantially alters chronic changes in brain metabolism induced by stroke.

Principle component analysis (PCA) was used to visually assess the separation between the experimental groups (Figure 2). There was both a separation along Component 1 due to treatment (Figure 2B \& C), and along Component 2 due to stroke (Figure 2B \& D). This analysis demonstrated that there was sub-clustering based on both compound treatment and disease state. A summary of the numbers of chemicals that achieved statistical significance $(p \leq 0.05)$, as well as whether they were increased or decreased is shown in Table 1, and a full list of the metabolic changes are provided in Supplementary Tables 1 and 2.

Next, we used a pathway surveyor tool to evaluate which key pathways are altered by stroke and by LM11A-31 treatment after stroke. This finding revealed that LM11A-31 can mitigate stroke induced decreases in the levels of multiple neurotransmitters, as well as stroke induced changes in redox homeostasis and glucose metabolism. With regard to neurotransmitter levels, we found that following stroke, mice had lower levels of glutamate, serotonin, and acetylcholine in the ipsilateral hemisphere compared to mice that underwent sham surgery (Figure 3A-C). Treatment 
with LM11A-31 after stroke significantly increased the levels of each of these neurotransmitters, except for glutamate. Notably, levels of dopamine trended towards a significant reduction following stroke $(p=0.1038)$ and were also significantly increased by LM11A-31 treatment (Figure 3D).

Regarding redox homeostasis, global metabolic profiling revealed that stroke compared to sham surgery resulted in a decrease in reduced glutathione (GSH) in the ipsilateral hemisphere. LM11A-31 restored GSH in stroked mice (Figure 4A). LM11A-31 also increased levels of oxidized glutathione (GSSG; Figure 4B). These results indicate that LM11A-31 treatment increases the pool of GSH available to modulate chronic changes in redox homeostasis caused by stroke.

Regarding glucose homeostasis (glycolysis pathway shown in Figure 5A), although levels of glucose were undetected, levels of pyruvate were reduced in the ipsilateral hemisphere of the mice that underwent stroke compared to sham surgery (Figure 5B). This finding indicates that there is a reduction in glycolysis in the ipsilateral hemisphere at 3 weeks after stroke. There was also a trend towards a reduction in acetyl-CoA, the oxidative decarboxylation product of pyruvate (Figure 5B). This finding is an indication that the reduction in glycolysis reduces the amount of acetyl-CoA available for the tricarboxylic acid (TCA) cycle. Treatment with LM11A-31 after stroke increased levels of acetyl-CoA (Figure 5B).

Treatment with LM11A-31 after stroke also altered the glycogenesis/glycogenolysis pathway (Figure 5C). This finding is supported by increases in maltotetraose, maltotriose, and maltose in the ipsilateral hemispheres of the LM11A-31 treated mice compared to the vehicle treated mice after stroke (Figure 5D). Like glucose, glycogen was undetected and so it is unclear whether these differences in intermediates are the result of increased glycogenolysis or increased glycogenesis. However, combined with the elevated level of acetyl-CoA seen in LM11A-31 treated 
mice compared to vehicle treated mice following stroke, these findings provide evidence that LM11A-31 treatment increases glycogenolysis, thereby increasing the amount of glucose available for acetyl-CoA generation.

Acetyl-CoA can also be generated by beta oxidation of long-chain fatty acids. To determine whether the increase in acetyl-CoA was the result of increased fatty acid oxidation we evaluated whether LM11A-31 treatment after stroke increased the levels of carnitine and acetyl-carnitine, key metabolites required for the generation of acetyl-CoA by beta oxidation. Although levels of carnitine and acetyl-carnitine were increased by stroke, they were not further increased by LM11A-31 treatment after stroke (Supplementary Tables 1 and 2). This result indicates that the increase in acetyl-CoA levels in response to LM11A-31 treatment after stroke is unlikely due to an increase in the beta oxidation of long-chain fatty acids.

\section{Phase I: Evaluation of cytokine/chemokine levels in the infarct in LM11A-31 treated mice}

We have previously provided evidence that stroke infarcts are sites of chronic inflammation (Doyle et al., 2015; Nguyen et al., 2016; Zbesko et al., 2018). To determine whether LM11A-31 has an effect on chronic inflammation in the infarct, we used a multiplex immunoassay to measure cytokines/chemokines within the infarcts of Phase I mice and tissue from the equivalent region of the cortex from sham operated mice. Analytes of interest included granulocyte colony-stimulating factor (G-CSF), granulocyte-macrophage colony-stimulating factor (GM-CSF), interferon- $\gamma$ (IFN$\gamma$ ), interleukin (IL)-1 $\alpha$, IL-1B, IL-2, IL-4, IL-5, IL-6, IL-7, IL-9, IL-10, IL-12(p40), IL-12(p70), IL-13, IL-15, IL-17, interferon-induced protein-10 (IP-10), keratinocytes-derived chemokine (KC), monocyte chemoattractant protein-1 (MCP-1), macrophage inflammatory protein-1 $\alpha$ (MIP-1 $\alpha$ ), macrophage inflammatory protein $1 \beta(\mathrm{MIP}-1 \beta)$, macrophage inflammatory protein-2 $\alpha$ (MIP-2 $\alpha$ ), 
regulated on activation normal T cell expressed and secreted (RANTES), and tumor necrosis factor- $\alpha$ (TNF- $\alpha)$. Out of these 25 cytokines/chemokines, only MIP-2 $\alpha$, IP-10, RANTES, and MIP$1 \beta$ were increased in the infarct site at 3 weeks after stroke when compared to tissue from the equivalent hemisphere of sham operated mice. LM11A-31 did not alter the levels of these cytokines/chemokines (Figure 6). These data indicate that two weeks of LM11A-31 treatment does not ameliorate or exacerbate inflammation within the infarct site at 3 weeks following stroke.

\section{Phase II: Evaluation of LM11A-31 on peripheral immune cell infiltration in the infarct and area of axonal degeneration}

The goal of Phase II was to evaluate the efficacy of LM11A-31 at ameliorating chronic neuropathology and neurodegeneration when treatment is initiated one week after stroke and after 12 weeks of daily dosing. The impact of LM11A-31 on inflammation in the infarct was determined by immunostaining of CD68+ microglia/macrophages, B220 $+\mathrm{B}$ cells, and CD3e+ T cells at the site of the infarct. Percent area occupied by CD68+, B220+, and CD3e+ immunostaining revealed that LM11A-31 treatment after stroke did not alter macrophage, B lymphocyte or T lymphocyte cell infiltration into the infarct at 13 weeks (Figure 7A-C).

We previously reported that in the weeks after stroke, CD3e+ T cells are not only present in the infarct, but are also present in the thalamus, striatum, and internal capsule in the ipsilateral hemisphere (Doyle et al., 2015). These are locations of degenerating axons that project from the sensorimotor cortex, which is the cortical region infarcted in the DH stroke model. Treatment with LM11A-31 did not affect T cell infiltration into the thalamic white matter tracts of the area of axonal degeneration (Figure 7D). 


\section{Phase II: Evaluation of the impact of LM11A-31 on secondary neurodegeneration}

Stroke causes delayed ipsilateral cortical atrophy and tissue thinning in 3-5-month-old C57BL/6 mice at 8 weeks post-stroke (Nguyen et al., 2018). This atrophy is more pronounced and results in enlargement of the ipsilateral lateral ventricle in 18-month-old mice (Nguyen et al., 2018). Therefore, we next sought to determine whether brain atrophy caused by stroke can be prevented or reduced by treatment with LM11A-31. As shown in the representative images, stroked mice that were treated with LM11A-31 demonstrated a significant reduction in the degree of post-stroke ventricular enlargement compared to those treated with vehicle (Figure 8A). Additionally, vehicle treated mice had significantly thinner ipsilateral primary somatosensory cortices compared to the contralateral cortices, and the equivalent cortices of LM11A-31-treated mice following stroke (Figure 8B). These data demonstrate that LM11A-31 can ameliorate atrophy of the ipsilateral hemisphere following stroke.

Stroke induced cortical atrophy correlates with neurodegeneration, as demonstrated by a reduction of neuronal nuclei marker, $\mathrm{NeuN}+$, in the peri-infarct cortex of mice following stroke (Chung et al., 2018; Zbesko et al., 2018). As depicted in the representative images and quantification of stroked mice treated with LM11A-31 compared to vehicle, LM11A-31 treatment attenuated this reduction in $\mathrm{NeuN}+$ immunoreactivity in the ipsilateral hemisphere (Figure $\mathbf{8 C}$, top images). Specifically, there was a reduction in $\mathrm{NeuN}+$ expression in the ipsilateral compared to the contralateral hemisphere of the stroked mice, and LM11A-31 administration resulted in a preservation of $\mathrm{NeuN}+$ immunoreactivity (Figure $\mathbf{8 C}$, bottom graphs). These data demonstrate that LM11A-31 can decrease the loss of NeuN+ immunoreactivity that occurs in the ipsilateral hemisphere in aged mice following stroke. 
Most of the cerebral cortex, such as the prefrontal and the motor cortices, along with the somatosensory cortex, are connected to basal forebrain cholinergic neurons (Wu et al., 2014; Gielow and Zaborszky, 2017). Brain regions with synaptic connections to ischemic regions can undergo transneuronal degeneration after stroke (Zhang et al., 2012; Chang et al., 2016), and previously we observed approximately $30 \%$ less ChAT+ staining in the medial septum of the basal forebrain of 18 -month-old C57BL/6 mice at 8 weeks post-stroke compared to sham surgery (Nguyen et al., 2018). In the present study, there was significantly more ChAT+ immunoreactivity in the medial septum of the mice treated with LM11A-31 compared to mice administered vehicle (Figure 8D).

Though primarily expressed in basal forebrain cholinergic neurons and pyramidal cortical and hippocampal neurons (Mufson EJ et al. 1992 PNAS 89:569-573; Miller MW and et al. 2000 Brain Res 852:355-366; Dougherty KD et al. 1999 J Neurosci 19:10140-10152; Zagrebelsky M et al. $2005 \mathrm{~J}$ Neurosci 25:9989-9999), p75 NTR is also present in neurons of the raphe nuclei, reticular formation, and locus coeruleus (noradrenergic neurons that contribute to the regulation of mood and behavioral states). $\mathrm{TH}+$ neurites of noradrenergic neurons in the rostral portion of the locus coeruleus innervate hippocampal and cortical areas, and are markedly affected in, and correlate with, severity of cognitive decline (Grudzien et al., 2007) in AD and other neurodegenerative diseases, such as Down syndrome, Parkinson's disease, and Huntington's disease (Iversen et al., 1983; Mann, 1988; Zweig et al., 1992; Zarow et al., 2003). To determine whether stroke results in a loss of $\mathrm{TH}+$ terminals innervating the peri-infarct cortical region, the density of $\mathrm{TH}+$ labeled projections fibers in the ipsilateral and contralateral cortex was quantified. As shown in Figure $8 \mathrm{E}$ (top images), $\mathrm{TH}+$ projection fibers appeared diminished in density within the ipsilateral primary somatosensory cortex, adjacent to the infarct, compared to the contralateral cortex in the stroked mice. There was a significant decrease in $\mathrm{TH}+$ expression in the ipsilateral compared to the 
contralateral hemisphere of the stroked mice and LM11A-31 administration did not attenuate this reduction (Figure 8E, bottom graph). Together, these data provide evidence that LM11A-31 can reduce brain atrophy and preserve $\mathrm{NeuN}+$ and $\mathrm{ChAT}+$ immunoreactivity following stroke but is less effective at preventing the loss of $\mathrm{TH}+$ projection fibers.

\section{Phase II: Evaluation of the effect of LM11A-31 on microglial activation and tau hyperphosphorylation}

Ser202 and Thr205, the epitopes recognized by the antibody AT8 when they are phosphorylated, are among the tau residues that contribute to the pathological conformations of p-tau (Jeganathan et al., 2008). Previously, we reported that 18-month-old stroked mice develop tau pathology in areas of degenerating white matter tracts following stroke (Nguyen et al., 2018). Furthermore, we have shown that excessive levels of $p$-tau+ staining are reduced in APP mutant mice after LM11A-31 treatment (Nguyen et al., 2014). Consequently, we determined whether LM11A-31 reduces p-tau accumulation in degenerating white matter tracts following stroke. As seen in Figure 9A (top images), quantitation revealed that (i) the ipsilateral hemisphere contained more $\mathrm{p}$-tau deposits than the contralateral hemisphere in the stroked mice, and (ii) treatment with LM11A-31, and not vehicle, attenuated this $\mathrm{p}$-tau burden within the ipsilateral hemisphere of stroked mice (Figure 9A, bottom right graph). A representative image from sections of APP mutant mice served as a p-tau/AT8 staining positive control (Figure 9A, bottom left image).

There is evidence for microglial activation in the proximity of tau deposits, and for the interplay of tau pathology and inflammatory cascades (Perea et al., 2020). Stroked mice exhibited numerous CD68+ immunoreactive cells, which displayed an activated microglia morphology (large cell 
bodies and bushy, thick processes) in the ipsilateral white matter tracks, and a more typical resting morphology (small cell body with long branching processes) in the contralateral white matter tracks. In mice that underwent stroke, LM11A-31 administration decreased the burden of activated microglia (Figure 9B, top images). Specifically, quantification of the area occupied by CD68+ labeled microglia/macrophages revealed significantly more CD68+ staining in the white matter tracks of the ipsilateral compared to the contralateral hemisphere of the stroked mice (Figure 9B, bottom graph). However, following stroke, LM11A-31 treated mice had less CD68+ staining than vehicle treated mice (Figure 9B, bottom graph). These findings demonstrate that LM11A-31 reduces microglial activation in thalamic white matter tracts in aged mice after stroke.

\section{Phase II: Evaluation of LM11A-31 on recovery following stroke}

Given the protective effects of LM11A-31 against brain atrophy, neurodegeneration, and loss of critical neurotransmitters, LM11A-31 was evaluated for its ability to improve recovery after stroke. Motor recovery was assessed by gait using the horizontal ladder rung test (Doyle et al., 2012). Previously, following DH stroke, C57BL/6 mice at 5 months of age displayed a motor impairment of the front limb contralateral to the stroke starting at day 1, which continues until 2 weeks postsurgery (Doyle et al., 2012).

As expected, 2 days following stroke, performance on the ladder rung test was impaired, which localized to the contralateral (left) front limb compared to naïve mice at baseline or pre-surgery (Figure 10A, left images: screenshots of video recordings to demonstrate a correct foot placement, and two different types of foot fault: an inability to grip the ladder rung with the front paw, and a foot slip). Scores from day 2 were used to stratify the mice into the LM11A-31 and vehicle treatment groups (Figure 10A, middle graphs: stratification method). At 2 weeks, 6 weeks, 
and 10 weeks following stroke, LM11A-31 treated mice performed better than vehicle treated mice on the ladder rung test, as measured by percent correct foot placement (Figure 10A, left graphs).

Building nests is an innate and spontaneous mouse behavior and nest construction can be assessed and used as a test for psychiatric disturbances that suggest a lack of motivation, anxiety and obsessive compulsive behaviors, or pain (Jirkof, 2014). Typically, mice will make nests achieving scores between 3.5 and 4.5, leaving $0.5 \mathrm{~g}$ of nestlets unshredded. Mice with hippocampal lesions achieve a median score of 2.0 (Deacon, 2006), which is in line with the score of our mice at 12 weeks following stroke (Figure 10B, left images). However, compared to treatment with vehicle alone, treatment with LM11A-31 significantly improved the performance of mice on the nest construction test following stroke (Figure 10B, right graph).

The $\mathrm{Y}$-maze was then used to test spontaneous alternation behavior (SAB). This test is based on the innate interest of rodents to explore a new environment. SAB has been shown to be impaired in $A D$, and Knowles and colleagues have demonstrated that LM11A-31 attenuates behavioral deficits on a Y-maze (Faizi et al., 2012; Knowles et al., 2013). A naïve or "normal" C57BL/6 mouse would typically score approximately 65-70\% SAB (Faizi et al., 2012; Knowles et al., 2013). However, following stroke, we did not detect a difference in SAB between stroked mice given LM11A-31 and those given vehicle (Figure 10C).

Novel object recognition (NOR) is a form of explicit memory known to be impaired in AD. The NOR test was used to evaluate whether LM11A-31 affects AD associated cognitive deficits in the stroke model. Following stroke there was no difference between stroked mice given LM11A-31 or vehicle; both experimental groups spent the same amount of time exploring a novel object relative to a familiar object (Figure 10D). 
The open field (OF) test provides a measure of anxiety and impulsivity. In a similar test of the anxiety-impulsivity spectrum, the light-dark transition test, aged, stroked mice display more impulsive risk-taking behavior (Nguyen et al., 2018). Here, we found a difference between stroked mice treated with LM11A-31 and those administered vehicle. Compared to vehicle treated mice, LM11A-31 treated mice entered the open center zone significantly less than the open zone around the perimeter. The vehicle treated mice displayed less cautious behavior, entering the center zone with greater frequency (Figure 10E). This provides evidence that treatment with LM11A-31 mitigates impulsive behavior in stroked mice. Together these data demonstrate that LM11A-31 can promote motor recovery, restore overall well-being, and mitigate impulsive like behavior in aged mice following stroke.

\section{DISCUSSION}

In this study, we asked whether LM11A-31, a small molecule modulator of $p 75^{\text {NTR }}$ signaling, could be used as a treatment for inhibiting neuronal degeneration, and thereby preserve neuronal health and function after stroke. Our rationale was that decreases in the ratio of neurotrophic versus neurodegenerative signaling play a critical role in other neurodegenerative diseases, such as AD, and studies using mouse models of AD have found therapeutic benefits from treatments targeting p75 NTR (Yang et al., 2008; Nguyen et al., 2014). LM11A-31 and its modulation of p75 ${ }^{\text {NTR }}$ signaling targets ubiquitous pathways and molecules shared among stroke and AD; for example, it can inhibit degenerative signaling via the tau kinases GSK3 $\beta$, calpain, cdk5, c-jun, and p38, and promote survival signaling via AKT and CREB.

Furthermore, there is evidence that pharmacological modulation of Trk, $\mathrm{p} 75^{\mathrm{NTR}}$, and NGF may prevent memory impairment in a rat model of global ischemia (Choucry et al., 2019), and that 
neurotrophins, such as BDNF may have therapeutic potential in protection against neurodegeneration and recovery from stroke and traumatic brain injury (Houlton et al., 2019). Following stroke, the expression of both pro-apoptotic and anti-apoptotic signaling molecules and transcription factors that are modulated by LM11A-31 are also upregulated in the ischemic penumbra (Yang et al., 2008; Nguyen et al., 2014; Simmons et al., 2016; Uzdensky, 2019; Yang et al., 2020a).

In the first phase of our study, we found that aged C57BL/6 male mice exhibit changes in brain metabolism following stroke that were attenuated after 2 weeks of oral administration of LM11A31. To ensure we were evaluating the impact of $L M 11 A-31$ on stroke recovery rather than on infarct size, treatment was delayed until 1 week after stroke. One of the most striking changes was in the levels of several neurotransmitters. Stroke induced significant decreases in levels of the neurotransmitter's glutamate, serotonin, and acetylcholine, and a trend towards a reduction in dopamine. However, the decreases in serotonin and acetylcholine were not seen in the LM11A31 treated stroked mice, which had significantly higher levels of these neurotransmitters than stroked mice treated with vehicle alone. LM11A-31 treated stroked mice also had significantly higher levels of dopamine than stroked mice treated with vehicle alone.

Serotonin's biological function is pleiotropic, as it modulates mood, cognition, reward, and learning and memory (Bacque-Cazenave et al., 2020). Acetylcholine is equally important, playing a role in attention, motivation, arousal, and memory (Sofuoglu and Mooney, 2009). The stroke induced reduction in levels of these essential neurotransmitters may impede proper signaling and thereby affect cognition, well-being, motor function, and psychological health. The preservation of the levels of these neurotransmitters and the increase in the levels of dopamine in response to LM11A-31 treatment suggests a broad improvement in neuronal health and function after stroke. 
In further support of this improvement, treatment with LM11A-31 for 12 weeks, with administration again delayed until 1 week after stroke, resulted in the preservation of brain volume and prevented loss of $\mathrm{NeuN}+$ immunoreactivity. Atrophy of the brain likely results from progressive degeneration of neurons, and the loss of these neurons likely impedes recovery and contributes to the manifestation of dementia after stroke in some patients.

LM11A-31 may preserve and optimize the function of some neurons better than others, as suggested by our finding that LM11A-31 reduced the loss of ChAT+ immunostaining, but not $\mathrm{TH}+$ immunostaining following stroke. This differential preservation may be explained by varying densities of $p 75^{\mathrm{NTR}}$ within the cell membranes of different neuronal subtypes; cholinergic neurons are rich in $p 75^{\mathrm{NTR}}$ and thus may bind more LM11A-31. Attesting to the protective effect of LM11A31 on cholinergic neurons, other studies have found that treatment with LM11A-31 attenuates cholinergic degeneration in AD mouse models (Knowles et al., 2013; Nguyen et al., 2014; Simmons et al., 2014), and more recently that it suppresses age related basal forebrain cholinergic neuronal degeneration (Xie et al., 2019).

Importantly, in conjunction with its effect on neuropathology following stroke, LM11A-31 also significantly attenuated impairments in motor function on the ladder rung test and significantly improved an activity of daily living in mice, as assessed by the nest construction test. Although the motor deficit induced by the stroke may have affected the ability of the mice to form nests, the improvement in nest building behavior nevertheless demonstrates that LM11A-31 treatment enhanced the ability of the mice to perform an innate behavior associated with their quality of living. LM11A-31 also significantly lessened stroke induced impulsive behavior in the open field test. Notably, in the open field test the vehicle treated mice entered the center zone of the open field arena with greater frequency than the LM11A-31 treated mice. This suggests that their motor 
disability was not a confound for this test. Together, our data show that the administration of a p75 ${ }^{\text {NTR }}$ ligand increases neurotransmitter levels, reduces chronic stroke neuropathology, and improves recovery after stroke.

With regard to the mechanisms by which LM11A-31 improved stroke outcome, although LM11A31 did not affect inflammation in the infarct, it did attenuate deficits in redox homeostasis following stroke by increasing the pool of reduced glutathione available to combat oxidative stress. Furthermore, treatment with LM11A-31 resulted in higher levels of acetyl CoA following stroke, suggesting LM11A-31 mitigated a stroke induced reduction in glycolysis. Although our data do not reveal the mechanism by which acetyl CoA levels were increased by LM11A-31 treatment, an increase in several key metabolites in the glycogenesis pathway suggest that it may be via glycogenolysis. However, further investigation into chronic changes in energy metabolism after stroke and how LM11A-31 treatment affects these changes is required.

We also determined that LM11A-31 treatment attenuated the accumulation of hyperphosphorylated tau in degenerating thalamic white matter tracts of aged mice following stroke. This finding is consistent with the demonstrated ability of LM11A-31 to inhibit the tau kinases glycogen synthase kinase 3 beta (GSK3 $\beta$ ), calpain, cyclin dependent kinase 5 (cdk5), cjun, and mitogen-activated protein kinase p38 (Yang et al., 2008; Nguyen et al., 2014; Yang et al., 2020b). Moreover, there is evidence for microglial activation in the proximity of tau deposits, and for an interplay between tau pathology and inflammatory cascades (Perea et al., 2020). Previous studies have found that LM11A-31 can attenuate increased microglial activation in APP mutant mice with $A \beta_{42}$ and p-tau deposition (Nguyen et al., 2014). In line with this evidence, we observed that LM11A-31 administration decreased microglial activation in the thalamic white matter tracts of aged mice following stroke. 
This study was designed with particular attention to STAIR recommendations (Lyden et al., 2021). Experiments were performed and analyzed in a randomized and blinded manner, we calculated the sample size based on a power analysis prior to the start of the study, and we considered the best route of administration for LM11A-31; oral delivery. We also chose a clinically useful therapeutic window by delaying treatment by 1 week, and selected an appropriate stroke model for evaluating post-stroke recovery rather than acute neuroprotection. However, there are further STAIR recommendations that should be followed prior to implementation of clinical testing. For example, follow up studies are necessary to determine if LM11A-31 can also improve recovery in a stroke model where there is recanalization and reperfusion to emulate patients treated with TPA and mechanical thrombectomy. LM11A-31 should also be tested by a second laboratory, in an alternative mouse strain (e.g., BALB/c mice), in a second species (e.g., rats), and in models that also feature common stroke comorbidities. It also needs to be ascertained if LM11A-31 is similarly effective in female mice. However, with regard to sex, LM11A-31 is similarly effective at reversing pathology in males and females in a mouse model of AD (Simmons et al., 2014), protects against neurodegeneration in both male and female HIV gp120 transgenic mice (Xie et al., 2021), and also protects against cisplatin induced peripheral neuropathy in both male and female mice (Friesland et al., 2014). Therefore, a sex difference is not expected.

A limitation of this study is that the precise mechanism by which LM11A-31 reduced brain atrophy, preserved $\mathrm{NeuN}+$ and ChAT+ immunoreactivity, and improved recovery following stroke is unclear. In AD, LM11A-31 prevents neuronal death, promotes neurotrophin signaling, and inhibits neurodegenerative signaling through the p75 ${ }^{\mathrm{NTR}}$. Although, there are distinct differences between stroke and $A D$, the loss of neurotrophic support in the brain is a fundamental neurodegenerative mechanism common to both diseases. However, follow up studies using p $75^{\text {NTR }}$ conditional 
knockout mice are necessary to determine the role of LM11A-31/p75 NTR signaling in cholinergic and other specific neuronal sub-populations following stroke.

In conclusion, the findings of this study support a promising role for $\mathrm{p} 75^{\mathrm{NTR}}$ as an effective target in the treatment of stroke beyond the acute setting, for which our current treatment options are extremely limited. Furthermore, because LM11A-31 has already reached Phase lla of clinical testing as a therapy for $A D$, once the STAIR recommendations for preclinical testing have been fulfilled, it could rapidly move to clinical trials in patients suffering from stroke and from patients suffering from $A D$ and stroke. 
Declarations of conflict of interest or financial disclosure: Dr. Longo has a financial interest in PharmatrophiX, a company focused on the development of LM11A-31. No other author has an actual of perceived conflict of interest with the contents of the article.

Acknowledgements: We are grateful to Metabolon for their help with design, data acquisition and data analysis of the metabolomic experiment.

\section{Authorship contributions:}

Participated in research design: TVN, FML, KPD

Conducted experiments: TVN, RHC, KEC, FGG, JCZ, MATG, DAB, HWM, SG, JBF

Contributed new reagents or tools: TY, FML

Performed data analysis: TVN, RHC, KEC, HWM, KPD

Contributed to the writing of the manuscript: TVN, RGS, FML, KPD 


\section{REFERENCES}

Bacque-Cazenave J, Bharatiya R, Barriere G, Delbecque JP, Bouguiyoud N, Di Giovanni G, Cattaert D and De Deurwaerdere P (2020) Serotonin in Animal Cognition and Behavior. Int J Mol Sci 21.

Becker K, Cana A, Baumgartner W and Spitzbarth I (2018) p75 Neurotrophin Receptor: A DoubleEdged Sword in Pathology and Regeneration of the Central Nervous System. Vet Pathol $55: 786-801$.

Chang SJ, Cherng JH, Wang DH, Yu SP, Liou NH and Hsu ML (2016) Transneuronal Degeneration of Thalamic Nuclei following Middle Cerebral Artery Occlusion in Rats. Biomed Res Int 2016:3819052.

Choi WT, Tosun M, Jeong HH, Karakas C, Semerci F, Liu Z and Maletic-Savatic M (2018) Metabolomics of mammalian brain reveals regional differences. BMC Syst Biol 12:127.

Choucry AM, Al-Shorbagy MY, Attia AS and El-Abhar HS (2019) Pharmacological Manipulation of Trk, p75NTR, and NGF Balance Restores Memory Deficit in Global Ischemia/Reperfusion Model in Rats. J Mol Neurosci 68:78-90.

Chung AG, Frye JB, Zbesko JC, Constantopoulos E, Hayes M, Figueroa AG, Becktel DA, Antony Day W, Konhilas JP, McKay BS, Nguyen TV and Doyle KP (2018) Liquefaction of the Brain following Stroke Shares a Similar Molecular and Morphological Profile with Atherosclerosis and Mediates Secondary Neurodegeneration in an OsteopontinDependent Mechanism. eNeuro 5.

Deacon RM (2006) Assessing nest building in mice. Nat Protoc 1:1117-1119.

Doyle KP, Fathali N, Siddiqui MR and Buckwalter MS (2012) Distal hypoxic stroke: a new mouse model of stroke with high throughput, low variability and a quantifiable functional deficit. $J$ Neurosci Methods 207:31-40. 
Doyle KP, Quach LN, Sole M, Axtell RC, Nguyen TV, Soler-Llavina GJ, Jurado S, Han J, Steinman L, Longo FM, Schneider JA, Malenka RC and Buckwalter MS (2015) B-lymphocytemediated delayed cognitive impairment following stroke. J Neurosci 35:2133-2145.

Faizi M, Bader PL, Saw N, Nguyen TV, Beraki S, Wyss-Coray T, Longo FM and Shamloo M (2012) Thy1-hAPP(Lond/Swe+) mouse model of Alzheimer's disease displays broad behavioral deficits in sensorimotor, cognitive and social function. Brain Behav 2:142-154.

Fogle JE, Hudson L, Thomson A, Sherman B, Gruen M, Lacelles BD, Colby BM, Clary G, Longo F and Meeker RB (2021) Improved neurocognitive performance in FIV infected cats following treatment with the p75 neurotrophin receptor ligand LM11A-31. J Neurovirol.

Franklin KBJ and Paxinos G (2008) The mouse brain in stereotaxic coordinates. Elsevier Science Amsterdam.

Friesland A, Weng Z, Duenas M, Massa SM, Longo FM and Lu Q (2014) Amelioration of cisplatininduced experimental peripheral neuropathy by a small molecule targeting p75 NTR. Neurotoxicology 45:81-90.

Gielow MR and Zaborszky L (2017) The Input-Output Relationship of the Cholinergic Basal Forebrain. Cell Rep 18:1817-1830.

Grudzien A, Shaw P, Weintraub S, Bigio E, Mash DC and Mesulam MM (2007) Locus coeruleus neurofibrillary degeneration in aging, mild cognitive impairment and early Alzheimer's disease. Neurobiol Aging 28:327-335.

Houlton J, Abumaria N, Hinkley SFR and Clarkson AN (2019) Therapeutic Potential of Neurotrophins for Repair After Brain Injury: A Helping Hand From Biomaterials. Front Neurosci 13:790.

Iversen LL, Rossor MN, Reynolds GP, Hills R, Roth M, Mountjoy CQ, Foote SL, Morrison JH and Bloom FE (1983) Loss of pigmented dopamine-beta-hydroxylase positive cells from locus coeruleus in senile dementia of Alzheimer's type. Neurosci Lett 39:95-100. 
James ML, Belichenko NP, Shuhendler AJ, Hoehne A, Andrews LE, Condon C, Nguyen TV, Reiser V, Jones P, Trigg W, Rao J, Gambhir SS and Longo FM (2017) [(18)F]GE-180 PET Detects Reduced Microglia Activation After LM11A-31 Therapy in a Mouse Model of Alzheimer's Disease. Theranostics 7:1422-1436.

Jeganathan S, Hascher A, Chinnathambi S, Biernat J, Mandelkow EM and Mandelkow E (2008) Proline-directed pseudo-phosphorylation at AT8 and PHF1 epitopes induces a compaction of the paperclip folding of Tau and generates a pathological (MC-1) conformation. J Biol Chem 283:32066-32076.

Jirkof $P$ (2014) Burrowing and nest building behavior as indicators of well-being in mice. $J$ Neurosci Methods 234:139-146.

Knowles JK, Rajadas J, Nguyen TV, Yang T, LeMieux MC, Vander Griend L, Ishikawa C, Massa SM, Wyss-Coray T and Longo FM (2009) The p75 neurotrophin receptor promotes amyloid-beta(1-42)-induced neuritic dystrophy in vitro and in vivo. J Neurosci 29:1062710637.

Knowles JK, Simmons DA, Nguyen TV, Vander Griend L, Xie Y, Zhang H, Yang T, Pollak J, Chang T, Arancio O, Buckwalter MS, Wyss-Coray T, Massa SM and Longo FM (2013) Small molecule p75NTR ligand prevents cognitive deficits and neurite degeneration in an Alzheimer's mouse model. Neurobiol Aging 34:2052-2063.

Longo FM and Massa SM (2008) Small molecule modulation of p75 neurotrophin receptor functions. CNS Neurol Disord Drug Targets 7:63-70.

Longo FM and Massa SM (2013) Small-molecule modulation of neurotrophin receptors: a strategy for the treatment of neurological disease. Nat Rev Drug Discov 12:507-525.

Lyden P, Buchan A, Boltze J, Fisher M and Consortium* SX (2021) Top Priorities for Cerebroprotective Studies-A Paradigm Shift: Report From STAIR XI. Stroke 52:30633071. 
Mann DM (1988) The pathological association between Down syndrome and Alzheimer disease. Mech Ageing Dev 43:99-136.

Massa SM, Xie Y, Yang T, Harrington AW, Kim ML, Yoon SO, Kraemer R, Moore LA, Hempstead BL and Longo FM (2006) Small, nonpeptide p75NTR ligands induce survival signaling and inhibit proNGF-induced death. J Neurosci 26:5288-5300.

Meeker RB, Poulton W, Clary G, Schriver M and Longo FM (2016) Novel p75 neurotrophin receptor ligand stabilizes neuronal calcium, preserves mitochondrial movement and protects against HIV associated neuropathogenesis. Exp Neurol 275 Pt 1:182-198.

Nguyen TV, Frye JB, Zbesko JC, Stepanovic K, Hayes M, Urzua A, Serrano G, Beach TG and Doyle KP (2016) Multiplex immunoassay characterization and species comparison of inflammation in acute and non-acute ischemic infarcts in human and mouse brain tissue. Acta Neuropathol Commun 4:100.

Nguyen TV, Hayes M, Zbesko JC, Frye JB, Congrove NR, Belichenko NP, McKay BS, Longo FM and Doyle KP (2018) Alzheimer's associated amyloid and tau deposition co-localizes with a homeostatic myelin repair pathway in two mouse models of post-stroke mixed dementia. Acta Neuropathol Commun 6:100.

Nguyen TV, Shen L, Vander Griend L, Quach LN, Belichenko NP, Saw N, Yang T, Shamloo M, Wyss-Coray T, Massa SM and Longo FM (2014) Small molecule p75NTR ligands reduce pathological phosphorylation and misfolding of tau, inflammatory changes, cholinergic degeneration, and cognitive deficits in $\operatorname{AbetaPP}(\mathrm{L} / \mathrm{S})$ transgenic mice. J Alzheimers Dis 42:459-483.

Perea JR, Bolos M and Avila J (2020) Microglia in Alzheimer's Disease in the Context of Tau Pathology. Biomolecules 10.

Rabinstein AA (2020) Update on Treatment of Acute Ischemic Stroke. Continuum (Minneap Minn) 26:268-286. 
Rha DW, Kang SW, Park YG, Cho SR, Lee WT, Lee JE, Nam CM, Han KH and Park ES (2011) Effects of constraint-induced movement therapy on neurogenesis and functional recovery after early hypoxic-ischemic injury in mice. Dev Med Child Neurol 53:327-333.

Ribbenstedt A, Ziarrusta H and Benskin JP (2018) Development, characterization and comparisons of targeted and non-targeted metabolomics methods. PLoS One 13:e0207082.

Seshadri S, Beiser A, Kelly-Hayes M, Kase CS, Au R, Kannel WB and Wolf PA (2006) The lifetime risk of stroke: estimates from the Framingham Study. Stroke 37:345-350.

Shi J, Longo FM and Massa SM (2013) A small molecule p75(NTR) ligand protects neurogenesis after traumatic brain injury. Stem Cells 31:2561-2574.

Simmons DA, Belichenko NP, Ford EC, Semaan S, Monbureau M, Aiyaswamy S, Holman CM, Condon C, Shamloo M, Massa SM and Longo FM (2016) A small molecule p75(NTR) ligand normalizes signalling and reduces Huntington's disease phenotypes in R6/2 and BACHD mice. Hum Mol Genet 25:4920-4938.

Simmons DA, Belichenko NP, Yang T, Condon C, Monbureau M, Shamloo M, Jing D, Massa SM and Longo FM (2013) A small molecule TrkB ligand reduces motor impairment and neuropathology in R6/2 and BACHD mouse models of Huntington's disease. J Neurosci 33:18712-18727.

Simmons DA, Knowles JK, Belichenko NP, Banerjee G, Finkle C, Massa SM and Longo FM (2014) A small molecule p75NTR ligand, LM11A-31, reverses cholinergic neurite dystrophy in Alzheimer's disease mouse models with mid- to late-stage disease progression. PLoS One 9:e102136.

Simmons DA, Mills BD, Butler lii RR, Kuan J, McHugh TLM, Akers C, Zhou J, Syriani W, Grouban M, Zeineh M and Longo FM (2021) Neuroimaging, Urinary, and Plasma Biomarkers of Treatment Response in Huntington's Disease: Preclinical Evidence with the p75(NTR) Ligand LM11A-31. Neurotherapeutics. 
Sofuoglu M and Mooney M (2009) Cholinergic functioning in stimulant addiction: implications for medications development. CNS Drugs 23:939-952.

Tep C, Lim TH, Ko PO, Getahun S, Ryu JC, Goettl VM, Massa SM, Basso M, Longo FM and Yoon SO (2013) Oral administration of a small molecule targeted to block proNGF binding to p75 promotes myelin sparing and functional recovery after spinal cord injury. $J$ Neurosci 33:397-410.

Uzdensky AB (2019) Apoptosis regulation in the penumbra after ischemic stroke: expression of pro- and antiapoptotic proteins. Apoptosis 24:687-702.

Wu H, Williams $\mathrm{J}$ and Nathans $\mathrm{J}$ (2014) Complete morphologies of basal forebrain cholinergic neurons in the mouse. Elife 3:e02444.

Xie Y, Meeker RB, Massa SM and Longo FM (2019) Modulation of the p75 neurotrophin receptor suppresses age-related basal forebrain cholinergic neuron degeneration. Sci Rep 9:5273.

Xie Y, Seawell J, Boesch E, Allen L, Suchy A, Longo FM and Meeker RB (2021) Small molecule modulation of the p75 neurotrophin receptor suppresses age- and genotype-associated neurodegeneration in HIV gp120 transgenic mice. Exp Neurol 335:113489.

Yang T, Knowles JK, Lu Q, Zhang H, Arancio O, Moore LA, Chang T, Wang Q, Andreasson K, Rajadas J, Fuller GG, Xie Y, Massa SM and Longo FM (2008) Small molecule, nonpeptide p75 ligands inhibit Abeta-induced neurodegeneration and synaptic impairment. PLoS One 3:e3604.

Yang T, Liu H, Tran KC, Leng A, Massa SM and Longo FM (2020a) Small-molecule modulation of the p75 neurotrophin receptor inhibits a wide range of tau molecular pathologies and their sequelae in P301S tauopathy mice. Acta Neuropathol Commun 8:156.

Yang T, Tran KC, Zeng AY, Massa SM and Longo FM (2020b) Small molecule modulation of the p75 neurotrophin receptor inhibits multiple amyloid beta-induced tau pathologies. Sci Rep 10:20322. 
Zarow C, Lyness SA, Mortimer JA and Chui HC (2003) Neuronal loss is greater in the locus coeruleus than nucleus basalis and substantia nigra in Alzheimer and Parkinson diseases. Arch Neurol 60:337-341.

Zbesko JC, Nguyen TV, Yang T, Frye JB, Hussain O, Hayes M, Chung A, Day WA, Stepanovic K, Krumberger M, Mona J, Longo FM and Doyle KP (2018) Glial scars are permeable to the neurotoxic environment of chronic stroke infarcts. Neurobiol Dis 112:63-78.

Zhang J, Zhang Y, Xing S, Liang Z and Zeng J (2012) Secondary neurodegeneration in remote regions after focal cerebral infarction: a new target for stroke management? Stroke 43:1700-1705.

Zweig RM, Ross CA, Hedreen JC, Peyser C, Cardillo JE, Folstein SE and Price DL (1992) Locus coeruleus involvement in Huntington's disease. Arch Neurol 49:152-156. 


\section{FOOTNOTES}

This work was supported by the National Institutes of Health National Institute of Neurological Disorders and Stroke [Grant R01NS096091], the National Institute on Aging [Grants R01AG063808 and R21AG062781], the United States Department of Veterans Affairs [Grant I01RX003224], the Jean Perkins Foundation and by the Leducq Foundation [Grant StrokeIMPaCT: Stroke - immune mediated pathways and cognitive trajectory]. 


\section{LEGENDS FOR FIGURES}

Figure 1. Study design for oral administration of $50 \mathrm{mg} / \mathrm{kg}$ LM11A-31 to aged mice as a treatment for stroke. (A) For Phase I (Cohort 1), mice were administered (Tx: treatment) LM11A31 or vehicle one time per day for a total of 2 weeks, beginning 1 week following stroke or sham surgery. At 3 weeks following surgery the infarct from each mouse was harvested for multiplex immunoassay. The remainder of each ipsilateral hemisphere was harvested for metabolomics. (B) For Phase II (Cohort 2), stroked mice were administered (Tx: treatment) LM11A-31 or vehicle 5 days per week for a total of 12 weeks, beginning 1 week following surgery. At 13 weeks following stroke surgery, brains were harvested for histology and immunostaining. In Phase II, mice also underwent behavioral testing at the indicated timepoints.

Figure 2. Principal component analysis (PCA) to allow for visual assessment of the clustering of each sample within each group. (A) Area dissected for global metabolic profiling. (B) PCA analysis with all four groups represented. (C) PCA reveals separation between vehicle and LM11A-31 treated mice along component 1 demonstrating a strong drug treatment effect. (D) PCA reveals separation between mice that were subjected to sham and stroke surgery along component 2, demonstrating a strong disease effect.

Figure 3. LM11A-31 attenuated stroke induced reductions in glutamate, serotonin, and acetylcholine, and increased levels of dopamine. Three weeks following stroke, the levels of (A) glutamate, (B) serotonin, and (C) acetylcholine were significantly reduced in the ipsilateral hemisphere compared to sham operated mice. The reductions in serotonin and acetylcholine were attenuated following 2 weeks of LM11A-31 (-31) compared to vehicle (veh) treatment. (D) Dopamine was not significantly reduced following stroke; however, it was significantly increased 
by 2 weeks of LM11A-31 compared to vehicle treatment. Group sizes: $n=5$ sham-veh, $n=5$ strokeveh, $n=6$ stroke-31. Data represent mean \pm SD. ${ }^{*} p<0.05$ and ${ }^{* *} p<0.01$.

Figure 4. LM11A-31 altered redox homeostasis after stroke. (A) Compared to sham operated mice, stroked mice had significantly lower levels of reduced glutathione (GSH) in the infarcted hemisphere. Stroked mice treated with LM11A-31 (-31) for 2 weeks had a significantly higher levels of GSH than stroked mice treated with vehicle (veh) alone, suggesting a prevention or reversal of the stroke induced decrease in GSH. (B) Levels of oxidized glutathione (GSSG) were also increased in LM11A-31-treated mice compared to vehicle treated mice. The increase in GSSG suggests there is a larger pool of GSH available to mitigate oxidative stress caused by the chronic sequelae of stroke. Group sizes: $n=5$ sham-veh, $n=5$ stroke-veh, $n=6$ stroke- 31 . Data represent mean $\pm S D .{ }^{*} p<0.05$, and ${ }^{* *} p<0.01$.

Figure 5. LM11A-31 altered glucose metabolism after stroke. (A) The glucose metabolism pathway or glycolysis. (B) Compared to sham operated mice, stroked mice had significantly lower levels of pyruvate (top graph) in their brains and showed a trend towards lower levels of acetylCoA $(p=0.0625 ;$ bottom graph). Although 2 weeks of LM11A-31 (-31) treatment did not significantly increase pyruvate levels compared to vehicle (veh) treatment, it did significantly increase levels of acetyl-CoA compared to vehicle treatment. (C) Glycogenolysis/Glycogenesis. (D) Although not altered by stroke, maltotetraose (top graph), maltotriose (middle graph), and maltose (bottom graph) were all increased following LM11A-31 treatment. This suggests that an increase in glycogenolysis may be the mechanism by which LM11A-31 increased acetyl-CoA after stroke. Group sizes: $n=5$ sham-veh, $n=5$ stroke-veh, $n=6$ stroke- 31 . Data represent mean \pm SD. ${ }^{*} p<0.05$ and ${ }^{* *} p<0.01$. 
Figure 6. LM11A-31 did not alter cytokine levels in the infarct. (A) Out of 25 cytokines measured by multiplex immunoassay, only MIP-2 $\alpha$, IP-10, RANTES, and MIP-1 $\beta$ were significantly increased in the infarct at 3 weeks following stroke compared to sham surgery. Treatment with LM11A-31 for 2 weeks, beginning 1 week after stroke did not significantly alter the levels of these cytokines. Group sizes: $n=5$ sham-veh, $n=5$ stroke-veh, $n=6$ stroke- 31 . Data represent mean \pm SD. ${ }^{*} p<0.05,{ }^{* *} p<0.01$, and ${ }^{* * *} p<0.001$

Figure 7. LM11A-31 did not alter immune cell infiltration in the infarct or $\mathrm{T}$ cell infiltration in the area of axonal degeneration. (A) Representative images and quantification of CD68 immunolabeled microglia and macrophages in the infarcts of mice given vehicle (veh) or LM11A31 (-31) for 12 weeks, starting 1 week post-stroke. Panels (i) and (ii) show the method of thresholding used to measure the \% area covered by cellular CD68+ immunostaining. Stroked mice given vehicle and stroked mice given LM11A-31 displayed similar CD68+ immunoreactivity; quantification revealed no significant differences between these treatment groups. (B) B220+ immunolabeled B cells and (C) CD3e+ immunolabeled T cells in the infarcts of mice given vehicle or LM11A-31 for 12 weeks, starting 1 week post-stroke. Stroked mice given vehicle and stroked mice given LM11A-31 also displayed similar B220+, and CD3e+ immunoreactivity; quantification revealed no significant differences between mice treated with either agent. (D) Representative images of $\mathrm{CD} 3 \mathrm{e}+$ immunolabeled $\mathrm{T}$ cells in white matter tracts projecting from the infarct area. There was no difference in CD3e+ immunoreactivity in stroked mice given vehicle or LM11A-31 in this area of axonal degeneration. Scale bars, $125 \mu \mathrm{m}$. Group sizes: $\mathrm{n}=10$ stroke-veh, $\mathrm{n}=10$ stroke-31. Data represent mean \pm SD.

Figure 8. LM11A-31 reduces brain atrophy and preserves NeuN and ChAT immunoreactivity following stroke. (A) Representative $2 \times$ images of Nissl stained whole brain 
sections from mice given vehicle (veh) or LM11A-31 (-31) for 12 weeks, starting 1 week poststroke (left images). Quantification of the lateral ventricle in the ipsilateral (ipsi) hemisphere revealed significant ventricle enlargement in stroked mice, which LM11A-31 significantly reduced (right graph). (B) Representative 2x images of cortical layers I-VI of the primary somatosensory cortex contralateral (contra) and ipsilateral (ipsi) to the infarct (left images). Quantification of cortical thickness in the ipsilateral compared to contralateral hemisphere revealed significant tissue thinness in stroked mice. Stroked mice treated with LM11A-31 showed significantly thicker tissue in this area than stroked mice treated with vehicle alone (right graph). Scale bar, $250 \mu \mathrm{m}$ (C) Representative $10 \times$ images of neuronal nuclei (NeuN) labeled in the ipsilateral primary somatosensory cortex (top images). Scale bar, $125 \mu \mathrm{m}$. Quantification revealed a significant reduction of NeuN+ staining in stroked mice, which was mitigated by LM11A-31 treatment (middle and bottom graphs). (D) Representative 10x images of choline acetyltransferase (ChAT)+ somas and neurites, and innervating projection fibers in the medial septum (top images). Many neurites (arrows) in the vehicle treated stroked mice displayed qualitative degenerative changes, including decreased length, which was not as evident in mice treated with LM11A-31. Scale bar, $125 \mu \mathrm{m}$. Quantification of cholinergic neurons, neurites, and fiber projections revealed a significant reduction of ChAT+ staining in stroked mice, which LM11A-31 also mitigated (bottom graph). (E) Representative $10 \times$ images of tyrosine hydroxylase $(\mathrm{TH})+$ projection fibers in the primary somatosensory cortex (top images and magnified outsets i-iii). Compared to sham operated mice, stroked mice displayed less abundant and more broken or disconnected fibers, which LM11A-31 treatment did not affect. Scale bar, $125 \mu \mathrm{m}$ (40 $\mu \mathrm{m}$ for outsets). $\mathrm{TH}+$ fiber quantification revealed significantly less $\mathrm{TH}+$ immunostaining in stroked mice compared to sham operated mice, which was not altered by LM11A-31 treatment (bottom graph). Group sizes: $n=10$ stroke-veh, $n=10$ stroke-31. Data represent mean \pm SD. ${ }^{*} p<0.05,{ }^{* *} p<0.01,{ }^{* *} p<0.001$, and ${ }^{* * *} p<0.0001$. 
Figure 9. LM11A-31 reduces tau pathology and microglial activation in white matter tracts of the thalamus after stroke. (A) Representative images of $p$-tau labelling using anti-tau phosphorylation ( $p$; AT8) in the thalamus (white matter tracts) of stroked mice treated with vehicle and LM11A-31 (top images). Scale bar, $125 \mu \mathrm{m}$. A representative image of $p$-tau labeling in the thalamus of aged hAPP-SL mice is provided as an AT8 staining positive control (bottom right image). Scale bar, $50 \mu \mathrm{m}$. Quantification revealed significantly more deposits of $p$-tau in stroked mice treated with vehicle compared to those treated with LM11A-31 (bottom left graph). (B) Representative 10x images of CD68 labeled microglia and macrophage in the thalamus (white matter tracts) in stroked mice treated with vehicle or LM11A-31 (top images). Scale bar, $125 \mu \mathrm{m}$. Quantification of CD68 immunoreactivity revealed significantly less staining in LM11A-31 treated stroked mice compared to vehicle treated stroked mice (bottom graph). Group sizes: $\mathrm{n}=10$ strokeveh, $n=10$ stroke-31. Data represent mean $\pm S D$. ${ }^{* * *} p<0.001$, and ${ }^{* * *} p<0.0001$.

Figure 10. LM11A-31 ameliorates stroke induced impairment of motor function, decline in overall well-being, and anxiety like behavior in aged mice. (A) Motor ability on the ladder rung test (left images show examples of correct foot placement and foot faults) was assessed at 1 week pre-surgery (left $\rightarrow$ first graph), and at 2 days (first graph), 2 weeks (third graph), 6 weeks (fourth graph), and 10 weeks (fifth graph) post-surgery. On Day 2, there was a motor deficit in stroke operated mice. Ladder rung test scores at 2 days post-surgery were used to stratify the stroked mice into treatment groups (second graph). Compared to vehicle treated mice, stroked mice given LM11A-31 displayed a significant improvement of motor function. (B) Mouse wellbeing was assessed with the nest construction test. Among stroked mice, LM11A-31 treated mice displayed significantly better nest building ability than mice given vehicle (left images and right graph). (C) In stroked mice, LM11A-31 treatment resulted in no significant differences in spatial working memory on the Y-maze. (D) LM11A-31 treatment in stroked mice also resulted in no 
significant differences in object recognition deficits, as assessed by the number of entries into the novel object zone (left graph) and the time spent in the novel object zone (right graph). (E) As assessed through an open field test, impulsive like behavior was significantly lower in LM11A-31 treated stroked mice than in vehicle treated stroked mice. Group sizes: $n=10$ stroke-veh, $n=10$ stroke-31. Data represent mean \pm SD. ${ }^{*} p<0.05,{ }^{* *} p<0.01,{ }^{* * *} p<0.0001$. 


\begin{tabular}{|c|c|c|c|c|}
\hline \multicolumn{5}{|c|}{ Statistical comparisons } \\
\hline $\begin{array}{c}\text { Group compared by } \\
\text { ANOVA }\end{array}$ & $\frac{\text { wt stroke veh }}{\text { wt sham veh }}$ & $\frac{\text { wt stroke LM11A-31 }}{\text { wt sham LM11A-31 }}$ & $\frac{\text { wt sham LM11A-31 }}{\text { wt sham veh }}$ & $\frac{\text { wt stroke LM11A-31 }}{\text { wt sham veh }}$ \\
\hline $\begin{array}{c}\text { Total biochemicals } \\
\text { p }<0.05\end{array}$ & 169 & 135 & 38 & 159 \\
\hline $\begin{array}{c}\text { Biochemicals } \\
(\uparrow \mid \downarrow)\end{array}$ & $128 \mid 41$ & $107 \mid 28$ & $23 \mid 15$ & $87 \mid 72$ \\
\hline
\end{tabular}

Table 1. Total numbers of biochemicals that significantly differed $(p \leq 0.05)$ between the listed experimental groups after global metabolic profiling from a total of 645 biochemicals analyzed. 


\section{Figure 1}

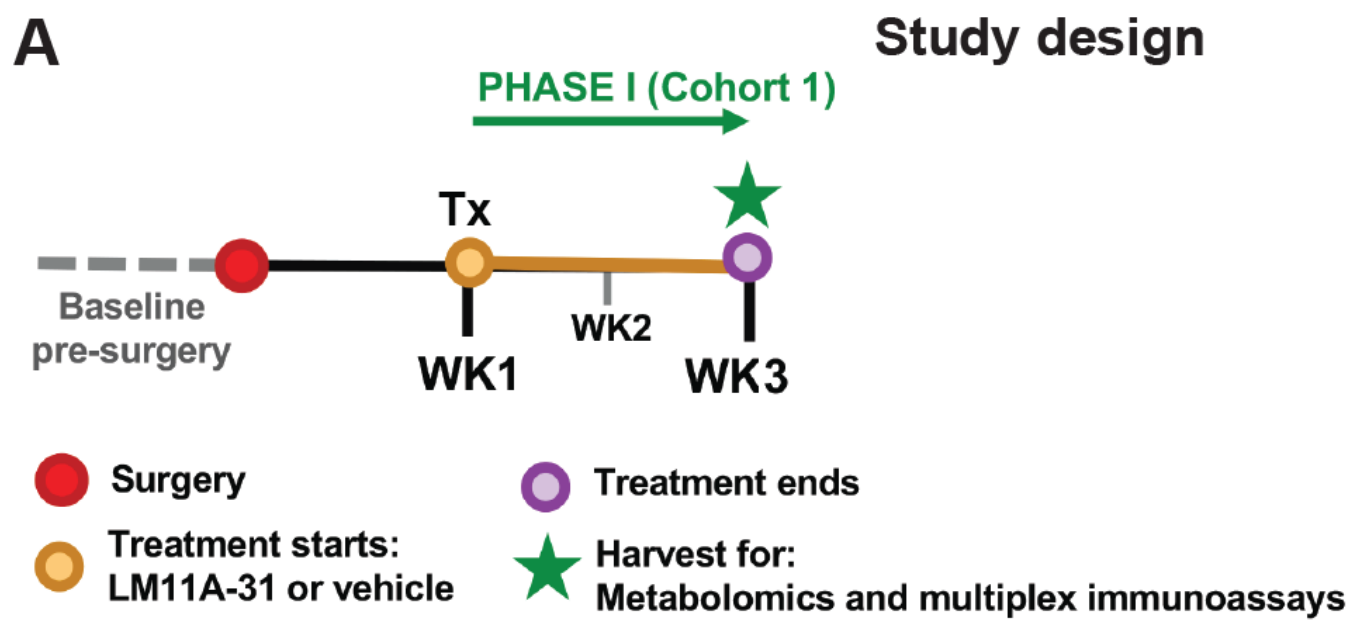

B

Study design

PHASE II (Cohort 2)

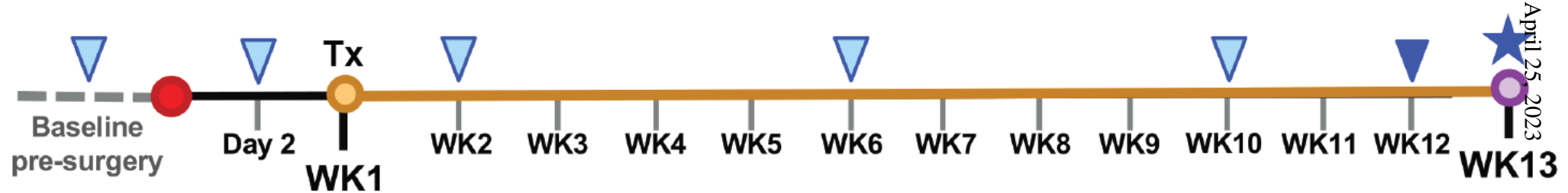

Surgery

Treatment starts:

LM11A-31 or vehicle
Treatment ends

$\nabla$ Behavior: Ladder rung
Behavior: Nest construction, Y-maze SAB, NOR, and OF

1 Harvest for:

Histology or immunostaining 
PETFast Eorwa Published on December 10, 2021 as DOI: 10.1124/jpet.121.000711

This art IC hat hersion may differ from this version.

A

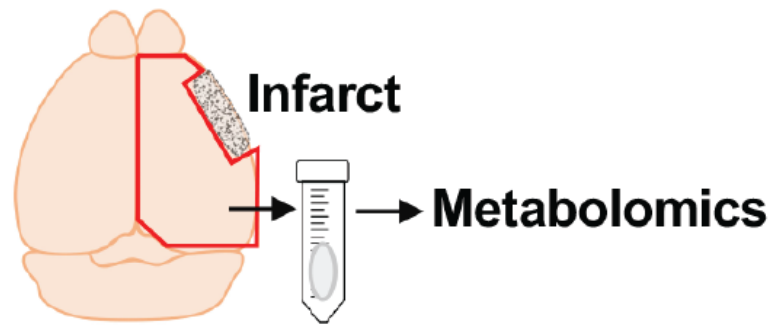

B PCA: All experimental groups

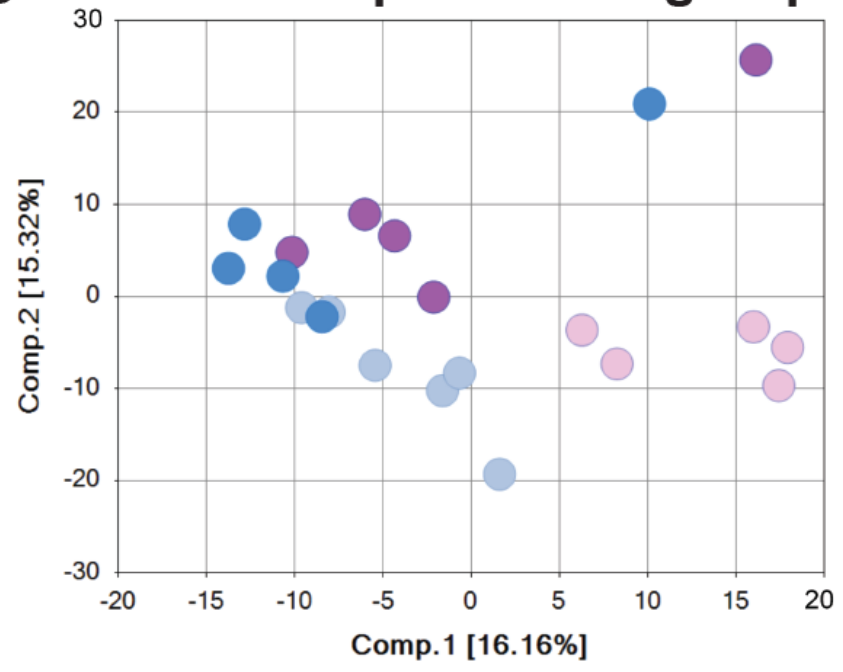

Sham-31

Sham-veh

Stroke-veh

Stroke-31

C PCA: Vehicle vs LM11A-31

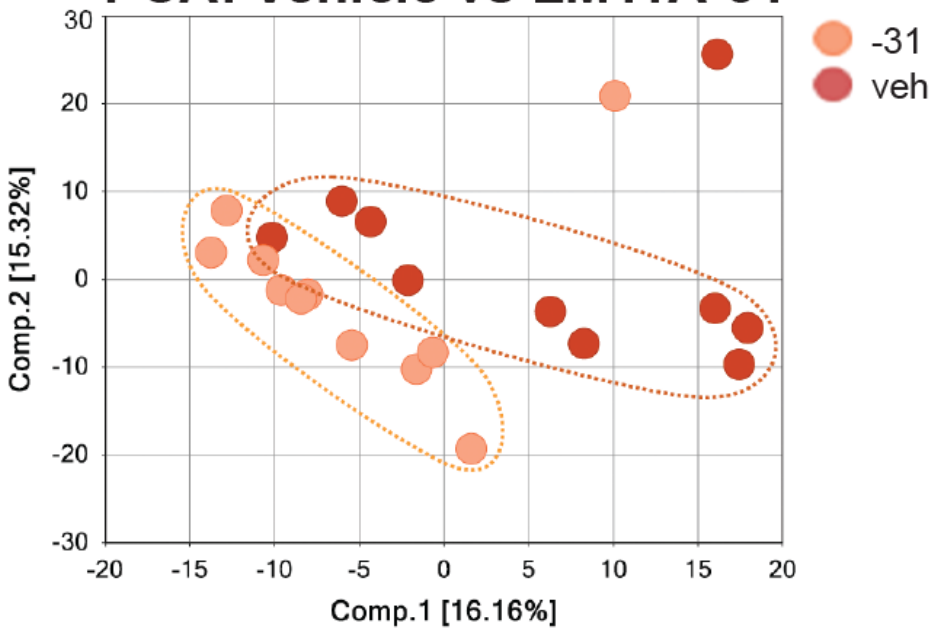

D PCA: Sham vs Stroke

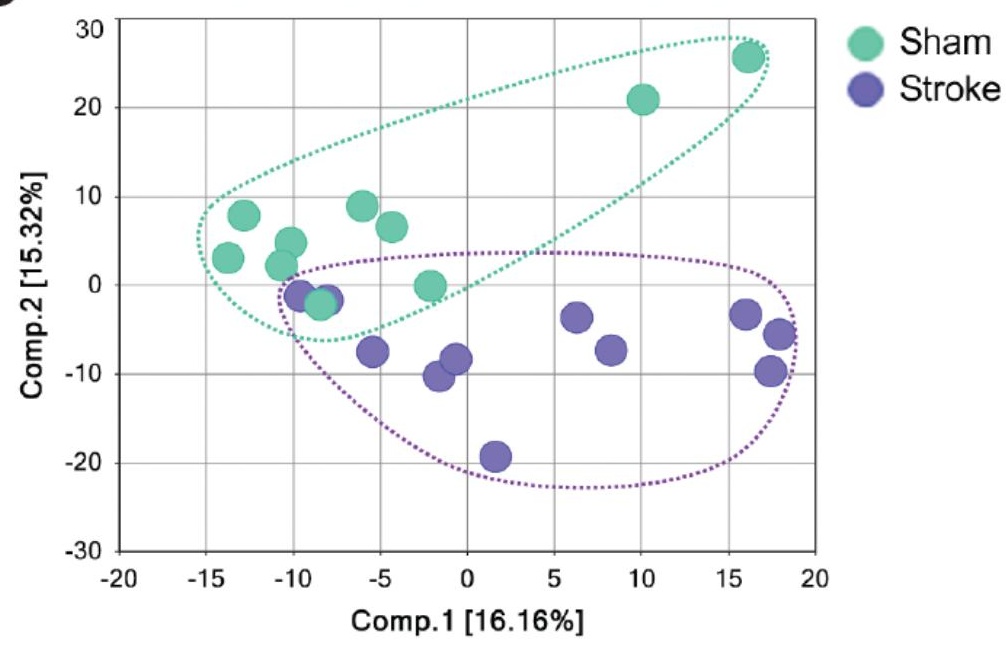


A

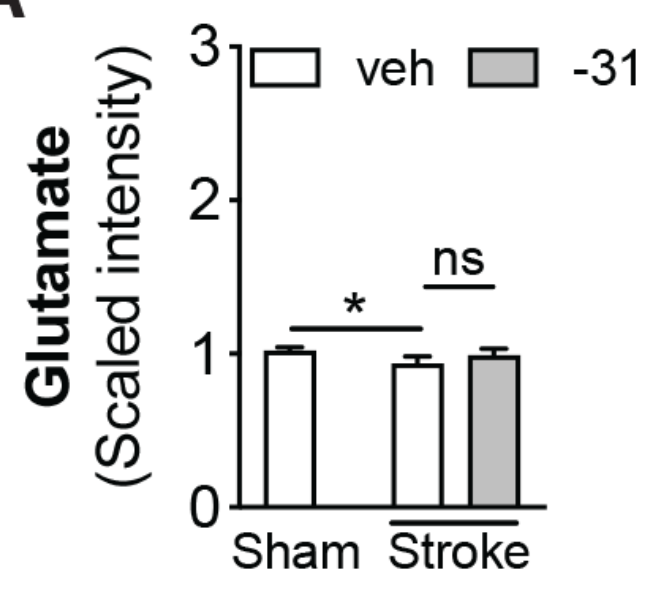

B

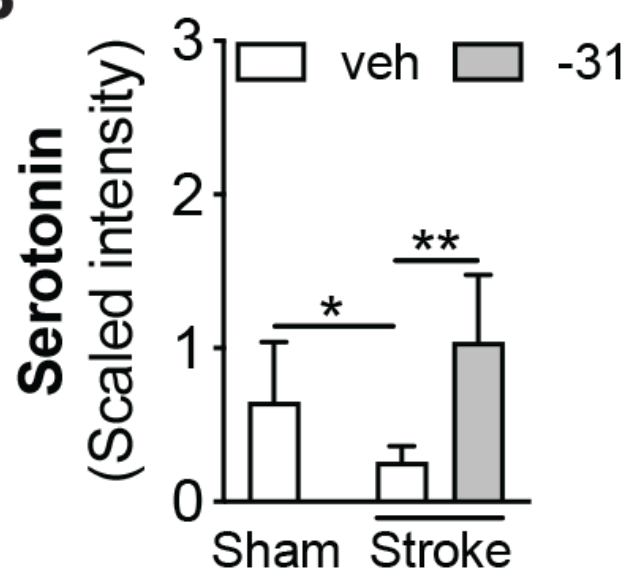

C

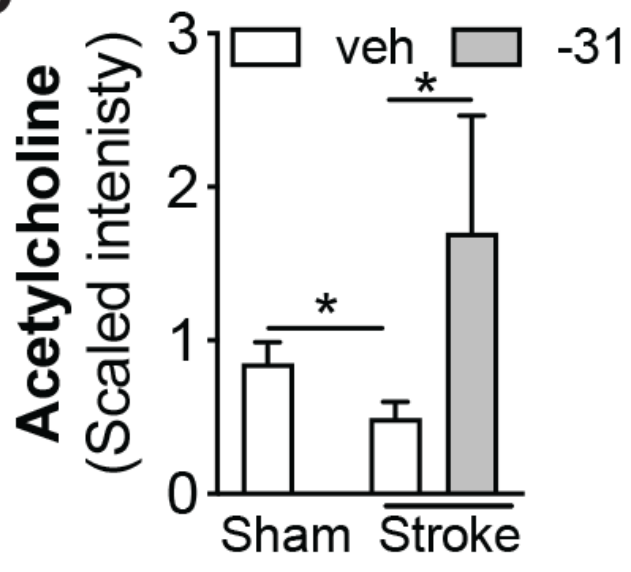

D

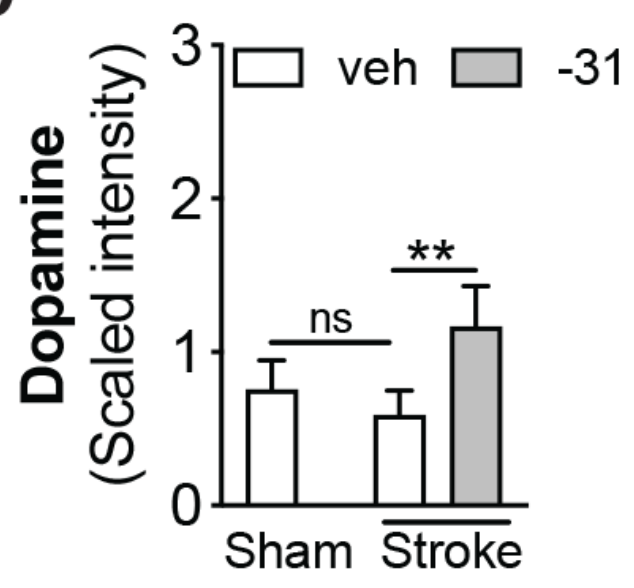



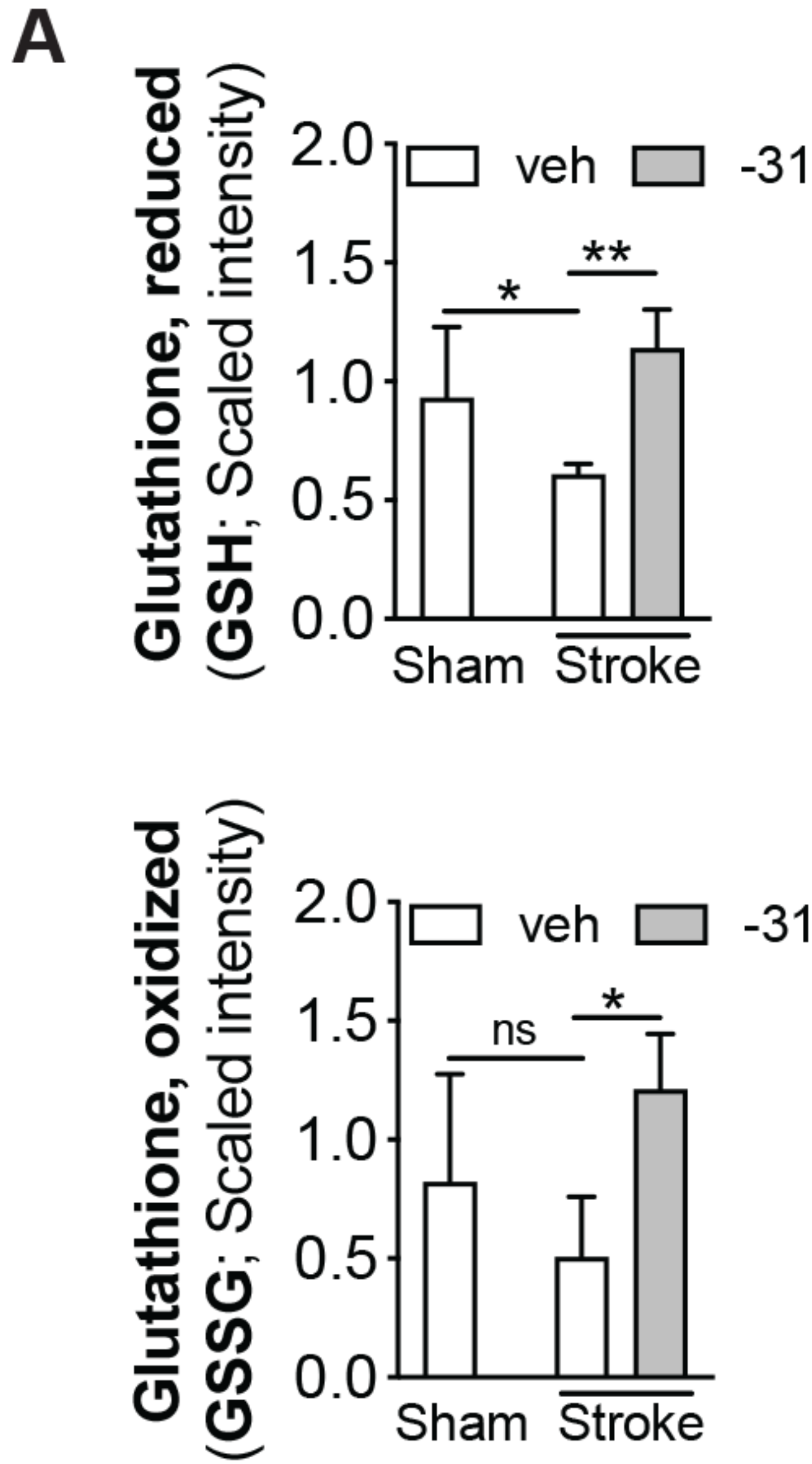

JPET Fast Forward. Published on December 10, 2021 as DOI: 10.1124/jpet.121.000711
Figure 5

A

\section{Glycolysis}

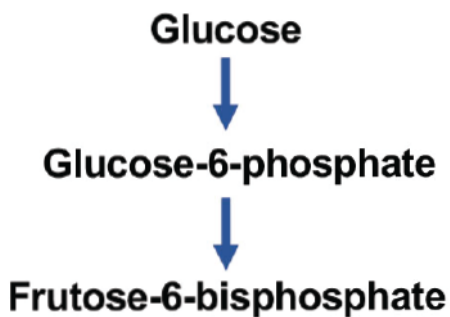

DHAP<smiles>CC1C=CC2CC1C2</smiles>

Glyceradehyde-3-phosphate

1,3-Biphosphoglycerate<smiles>[Te]C1CC1</smiles>

Glycerate/3-Phosphoglycerate

2-Phosphoglycerate

Phosphoenolpyruvate (PEP)

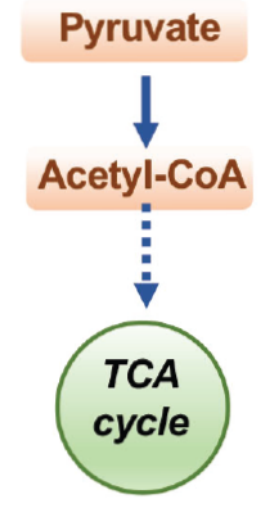

C

Glycogenolysis/Glycogenesis

Glucose
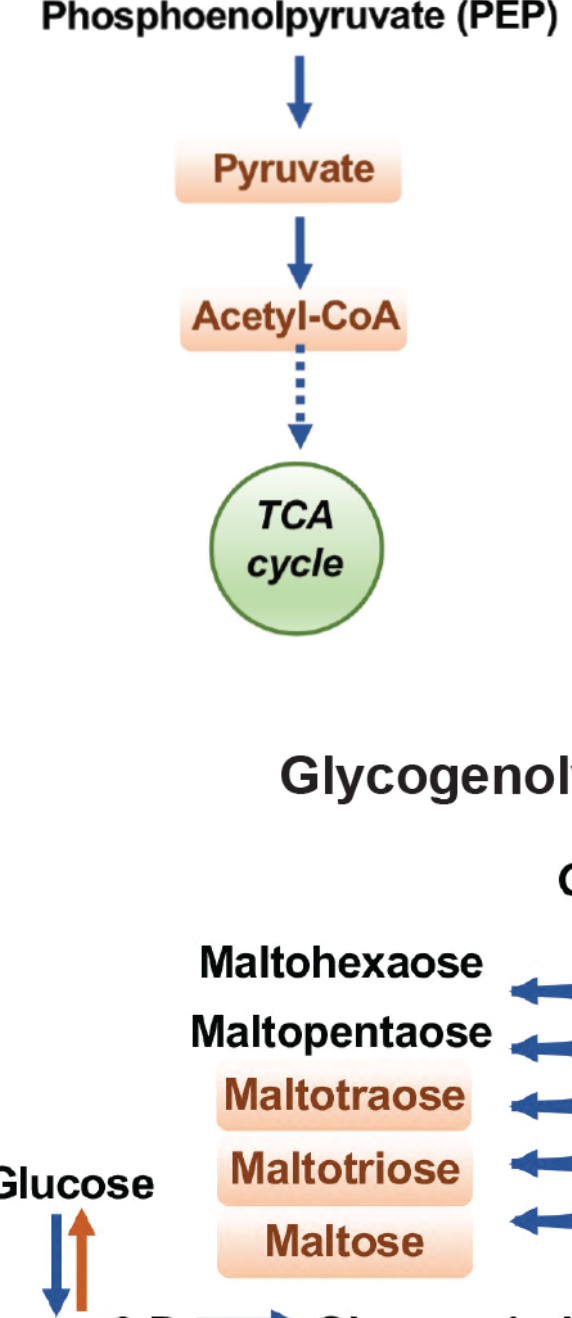

Glucose 6-P $\rightleftarrows$ Glucose-1 phosphate
B
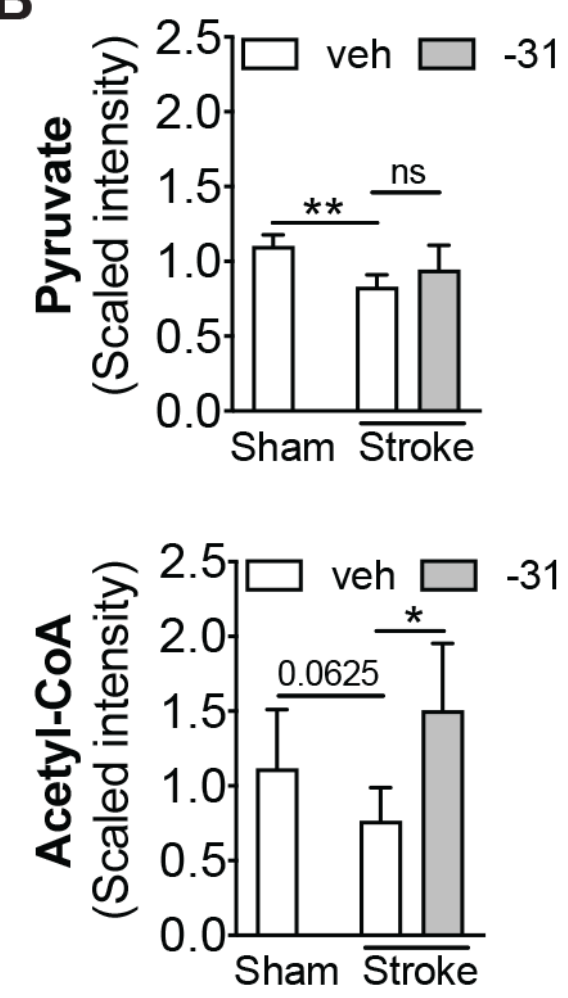

D
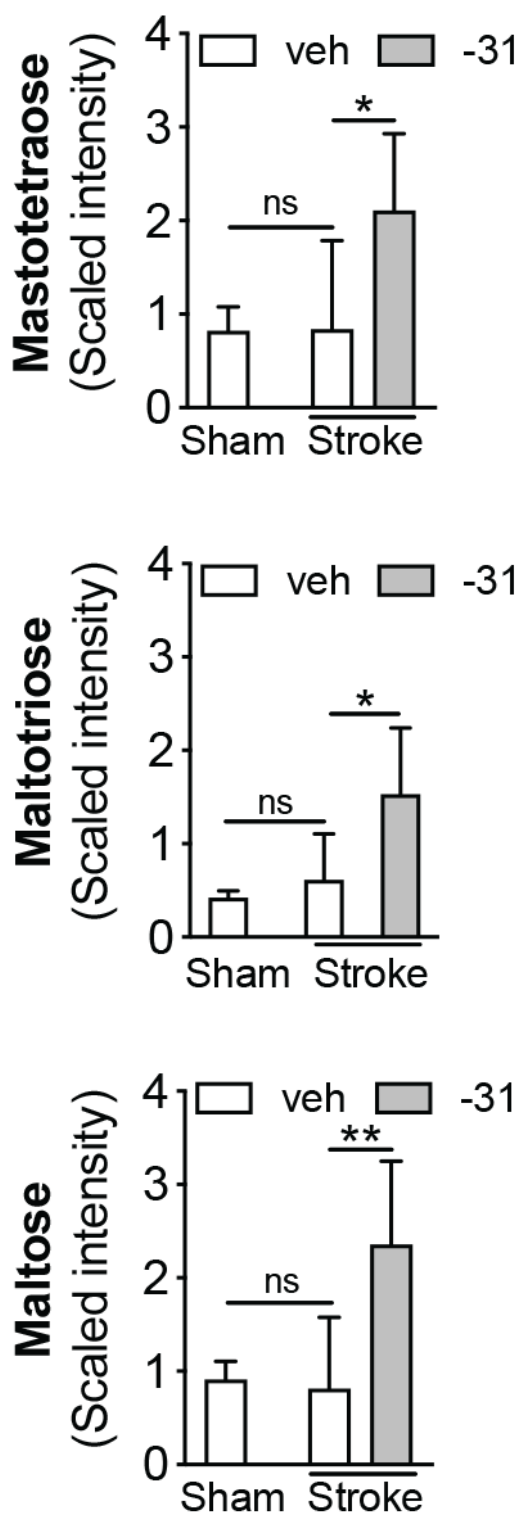


\section{Figure 6}
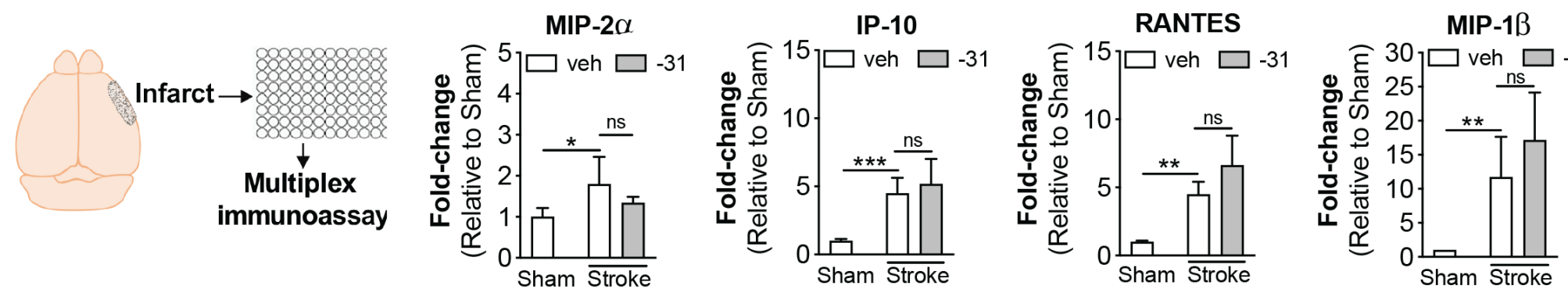


\section{Figure 7}

A

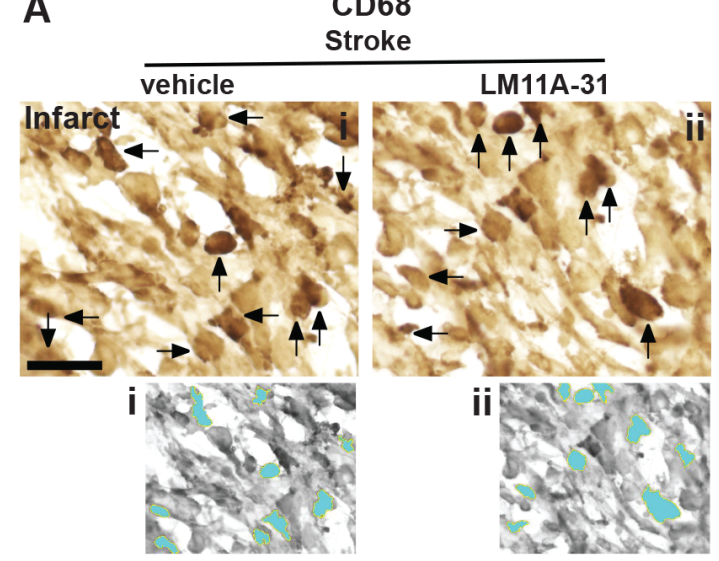

C
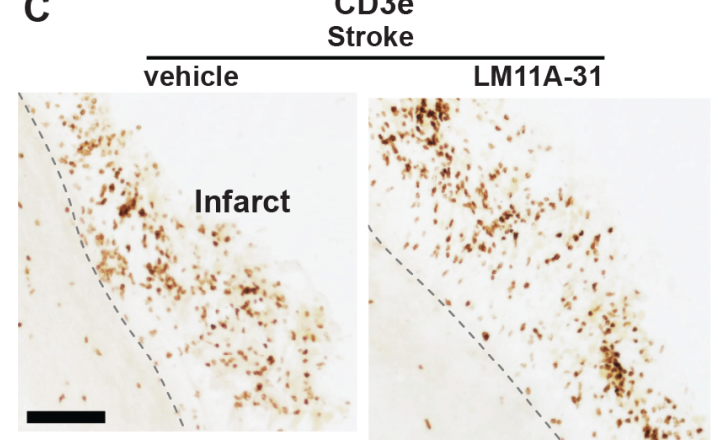

B
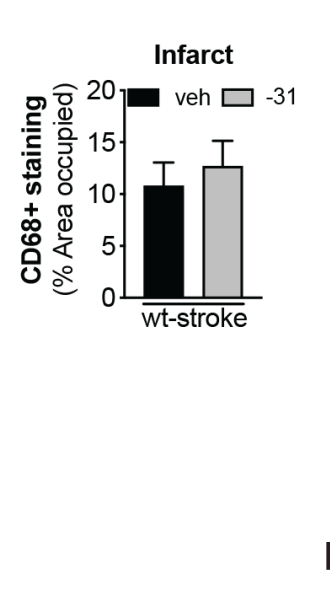

D

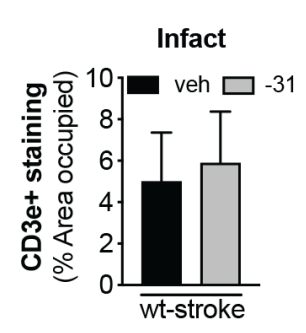

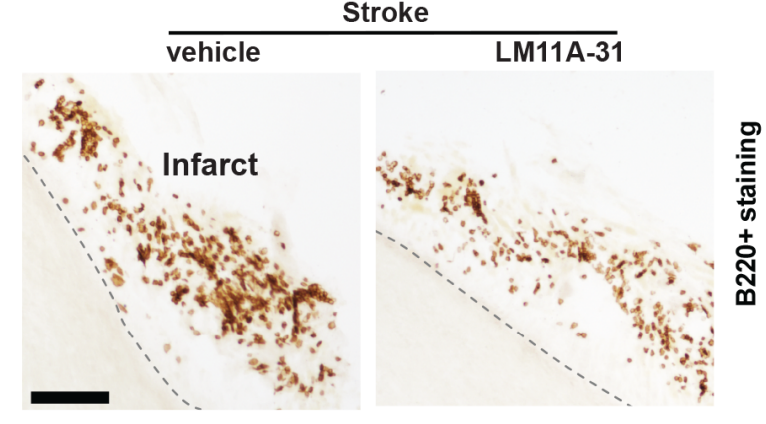

B220
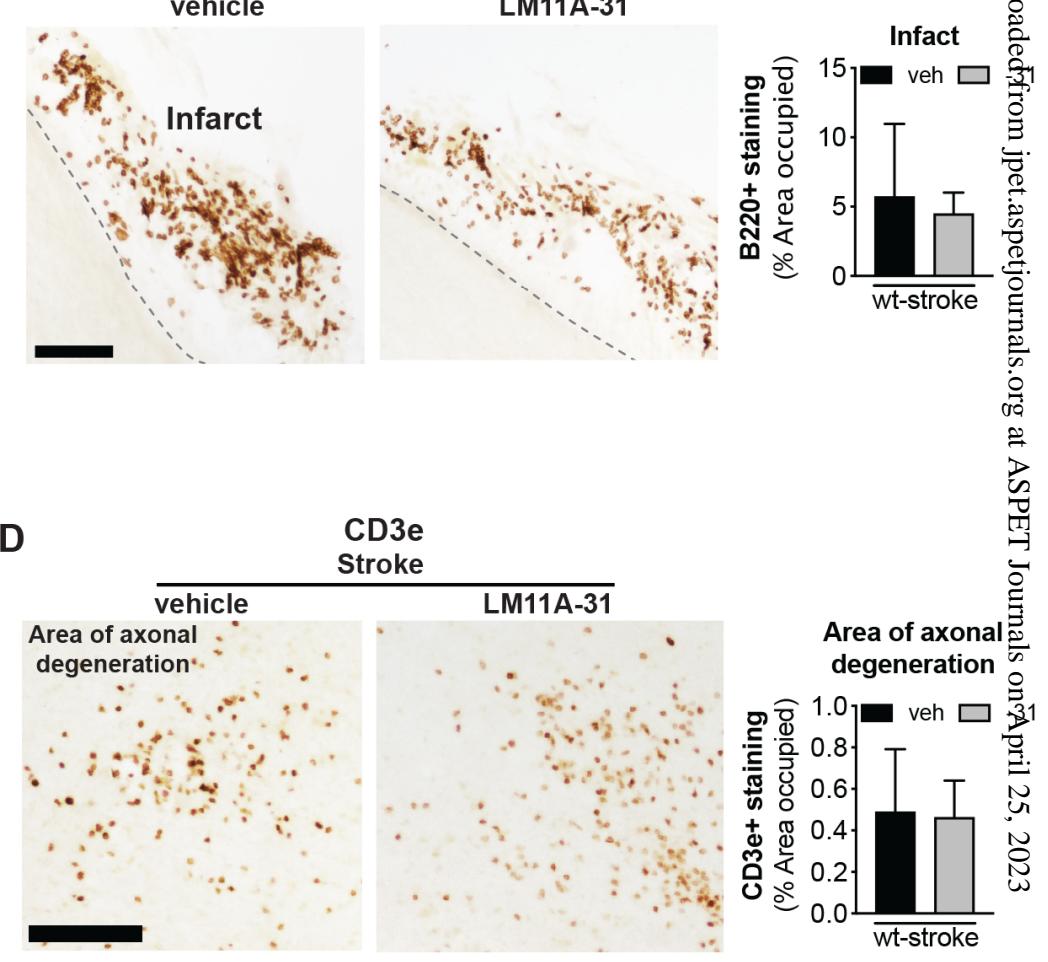


\section{Figure 8}

A

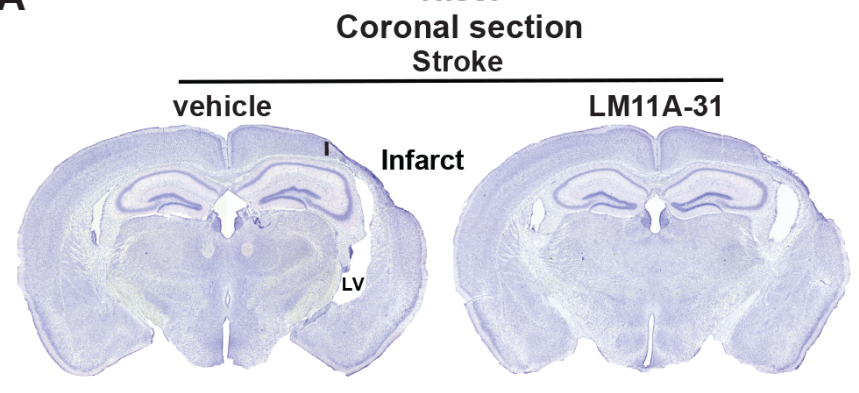

Nissl

nal section

B

Cortex I-VI

Stroke

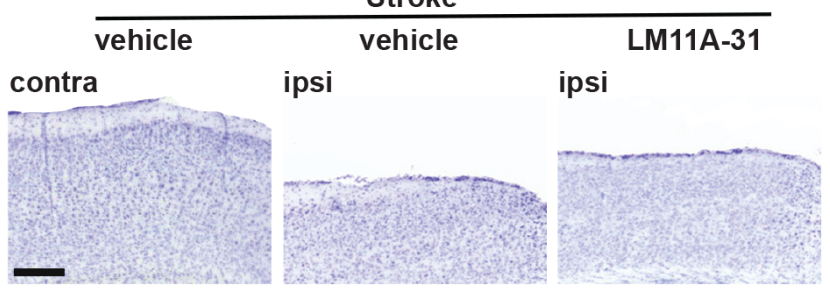

C

\begin{tabular}{lll} 
& $\begin{array}{c}\text { Cortex } \\
\text { Stroke }\end{array}$ & \\
\hline vehicle & vehicle & LM11A-31
\end{tabular}

Cortex

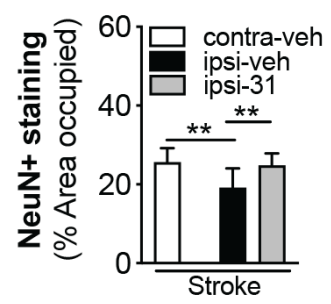

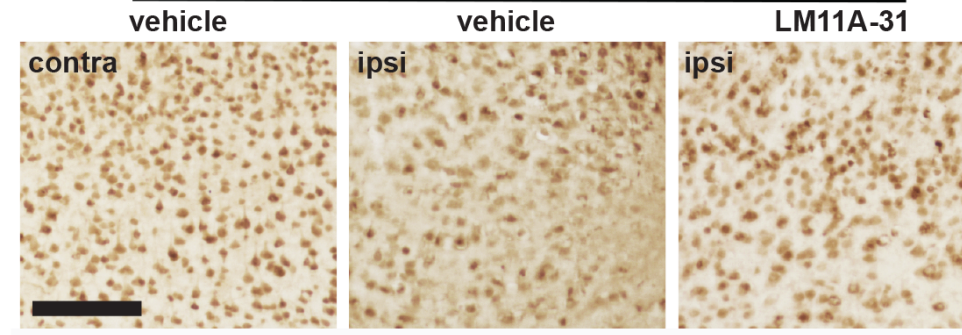
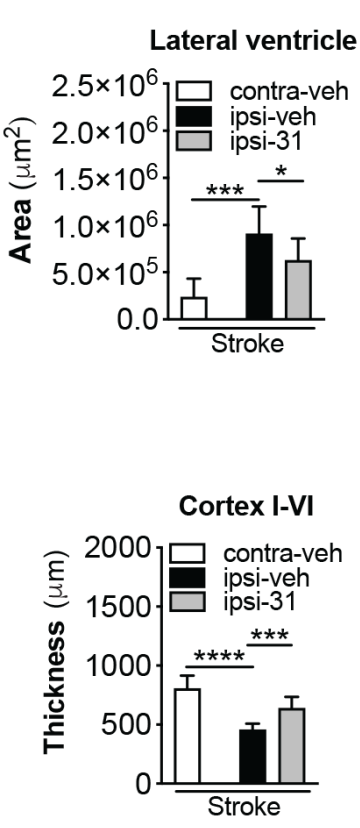

D

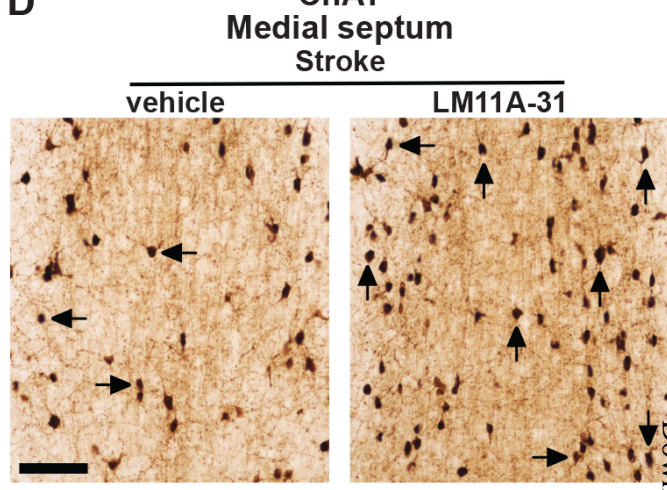

Medial spetum

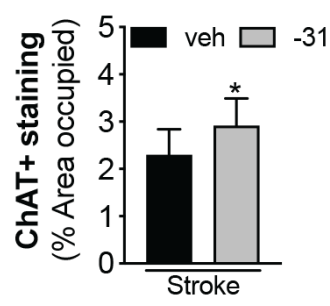

E

Tyrosine hydroxylase Cortex Stroke
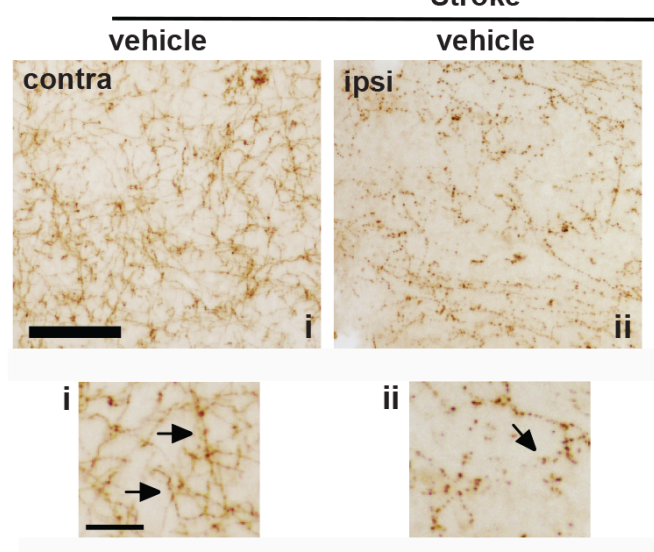

ii

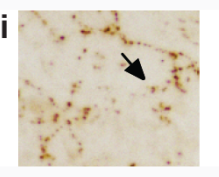

Cortex

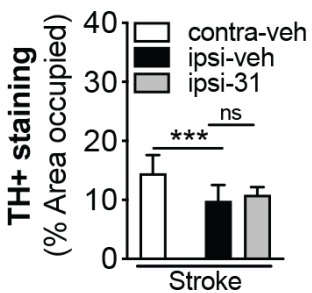


FiqurbET Fast Forward. Published on December 10, 2021 as DOI: 10.1124/jpet.121.000711
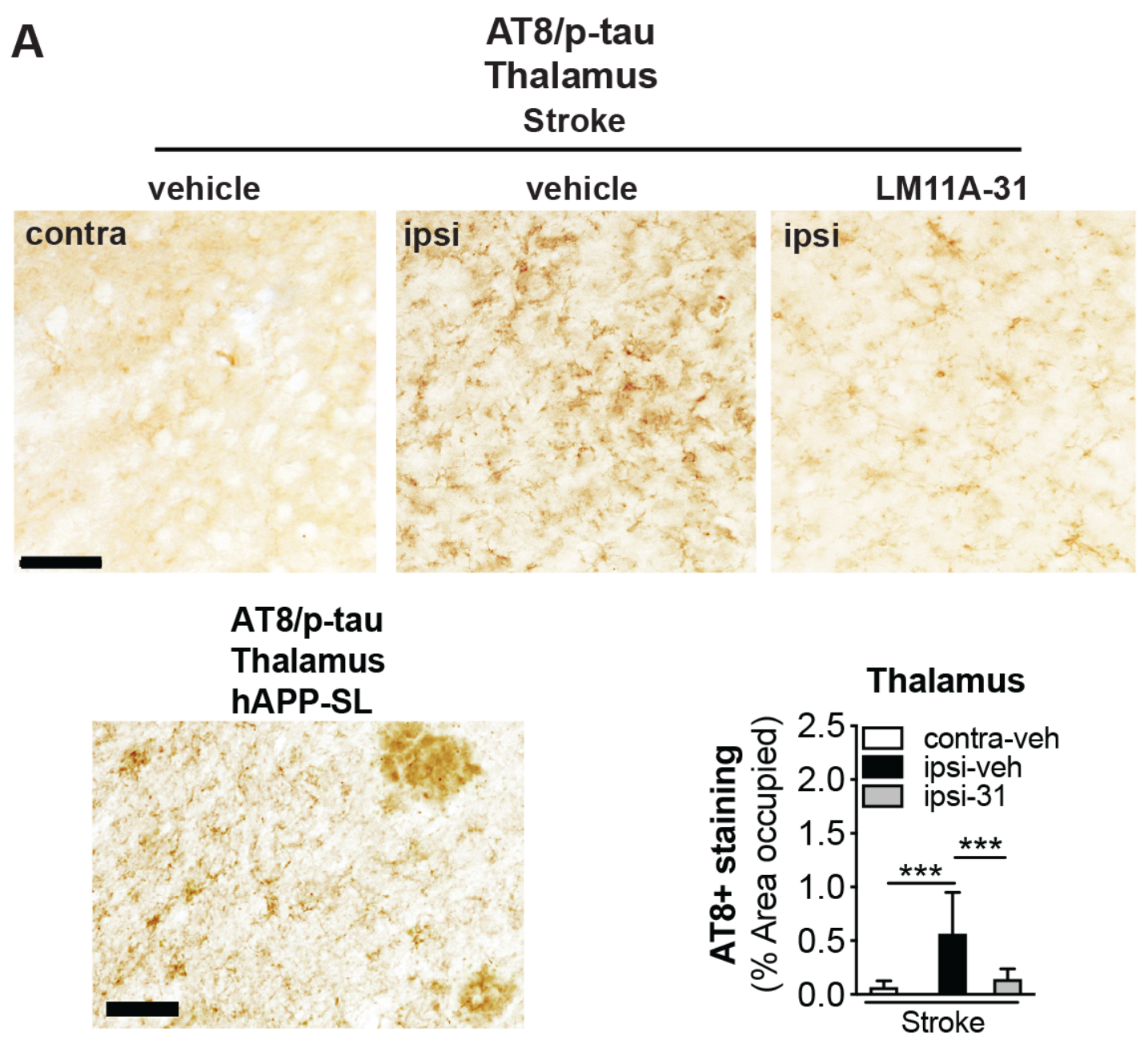

B

CD68

Thalamus

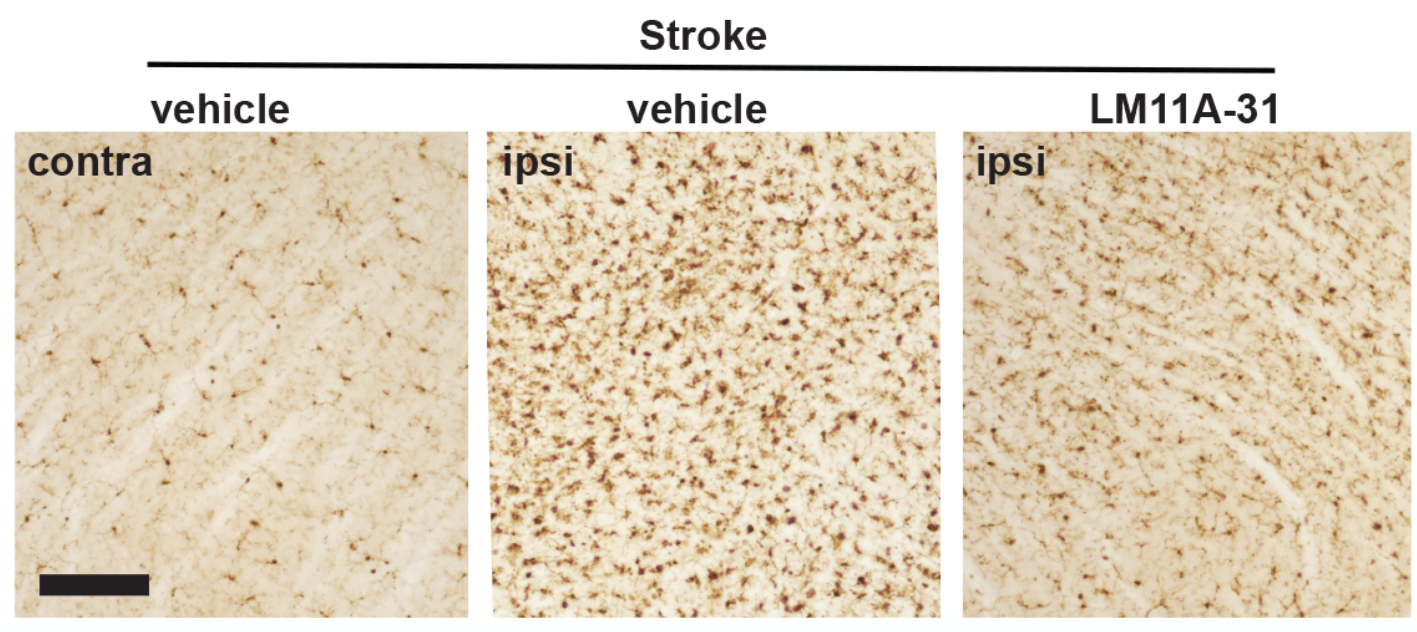

Thalamus

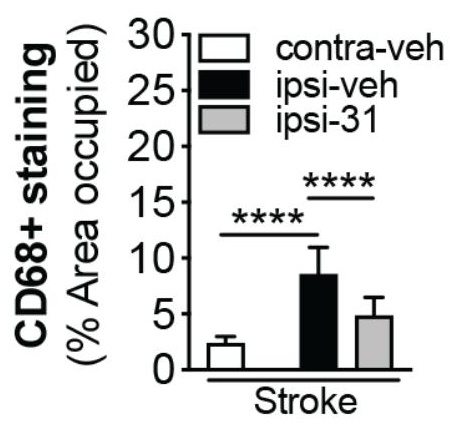




\section{Figure 10}

A

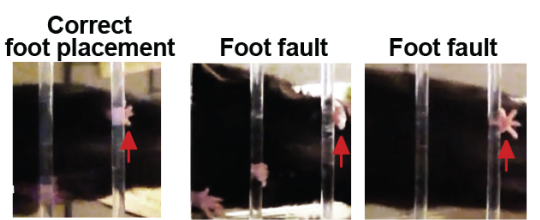

B

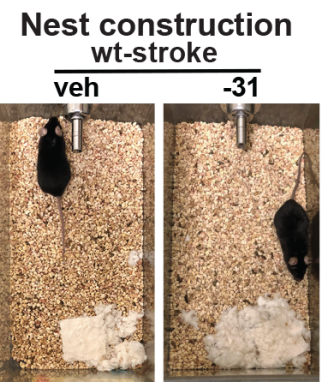

Nest construction wt-stroke

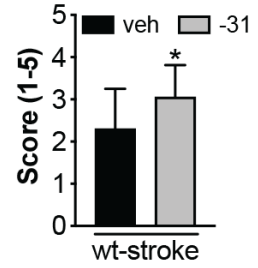

Ladder rung

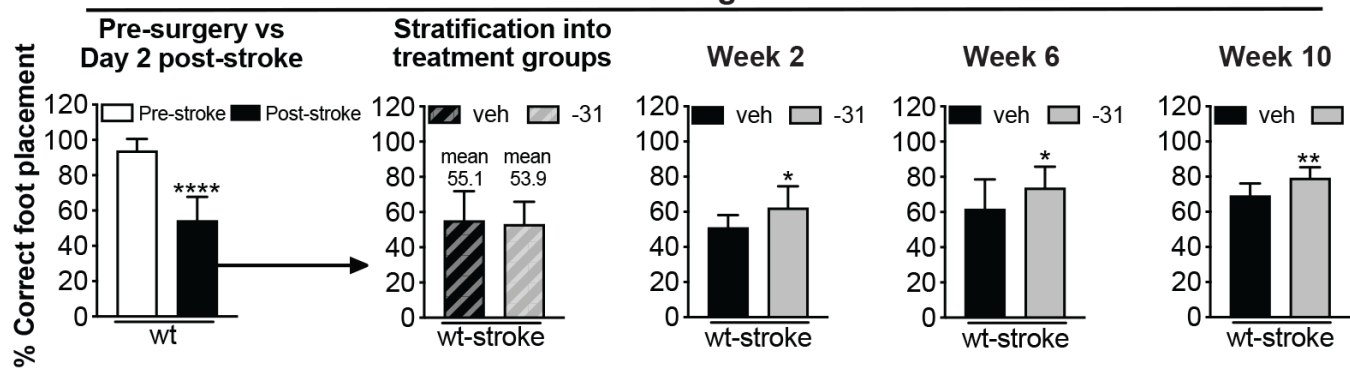

C

Y-maze

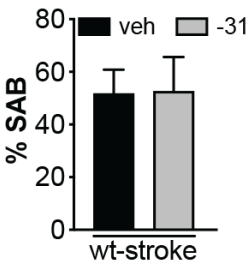

D

Novel object recognition

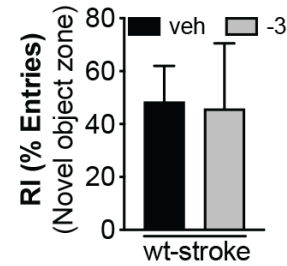

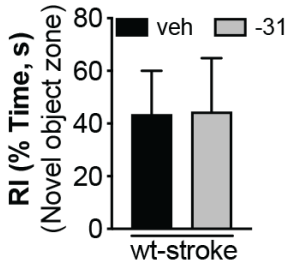

$\mathbf{E}$

Open field

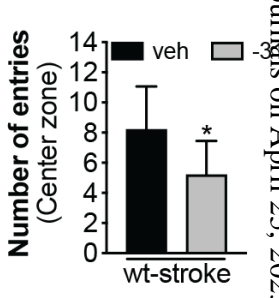

